\title{
Proteomics identifies neddylation as a potential therapy target in small intestinal neuroendocrine tumors
}

\author{
Omid Fotouhi ${ }^{1} \cdot$ Hanna Kjellin ${ }^{2} \cdot$ C. Christofer Juhlin $\mathbb{D}^{1,3} \cdot$ Yanbo Pan ${ }^{1,4} \cdot$ Mattias Vesterlund $\mathbb{D}^{1,4} \cdot$ Mehran Ghaderi $^{1}$. \\ Abdelhamid Yousef ${ }^{5} \cdot$ Hillevi Andersson-Sand ${ }^{4} \cdot$ Pedram Kharaziha $^{6} \cdot$ Stefano Caramuta $^{7} \cdot$ Magnus Kjellman $^{2,8}$. \\ Jan Zedenius ${ }^{2,8} \cdot$ Catharina Larsson $^{1} \cdot$ Lukas M. Orre ${ }^{1,4}$
}

Received: 26 October 2018 / Revised: 30 April 2019 / Accepted: 15 May 2019 / Published online: 12 August 2019

(c) The Author(s) 2019. This article is published with open access

\begin{abstract}
Patients with small intestinal neuroendocrine tumors (SI-NETs) frequently develop spread disease; however, the underlying molecular mechanisms of disease progression are not known and effective preventive treatment strategies are lacking. Here, protein expression profiling was performed by HiRIEF-LC-MS in 14 primary SI-NETs from patients with and without liver metastases detected at the time of surgery and initial treatment. Among differentially expressed proteins, overexpression of the ubiquitin-like protein NEDD8 was identified in samples from patients with liver metastasis. Further, NEDD8 correlation analysis indicated co-expression with RBX1, a key component in cullin-RING ubiquitin ligases (CRLs). In vitro inhibition of neddylation with the therapeutic agent pevonedistat (MLN4924) resulted in a dramatic decrease of proliferation in SINET cell lines. Subsequent mass spectrometry-based proteomics analysis of pevonedistat effects and effects of the proteasome inhibitor bortezomib revealed stabilization of multiple targets of CRLs including p27, an established tumor suppressor in SI-NET. Silencing of NEDD8 and RBX1 using siRNA resulted in a stabilization of p27, suggesting that the cellular levels of NEDD8 and RBX1 affect CRL activity. Inhibition of CRL activity, by either NEDD8/RBX1 silencing or pevonedistat treatment of cells resulted in induction of apoptosis that could be partially rescued by siRNA-based silencing of p27. Differential expression of both p27 and NEDD8 was confirmed in a second cohort of SI-NET using immunohistochemistry. Collectively, these findings suggest a role for CRLs and the ubiquitin proteasome system in suppression of p27 in SI-NET, and inhibition of neddylation as a putative therapeutic strategy in SI-NET.
\end{abstract}

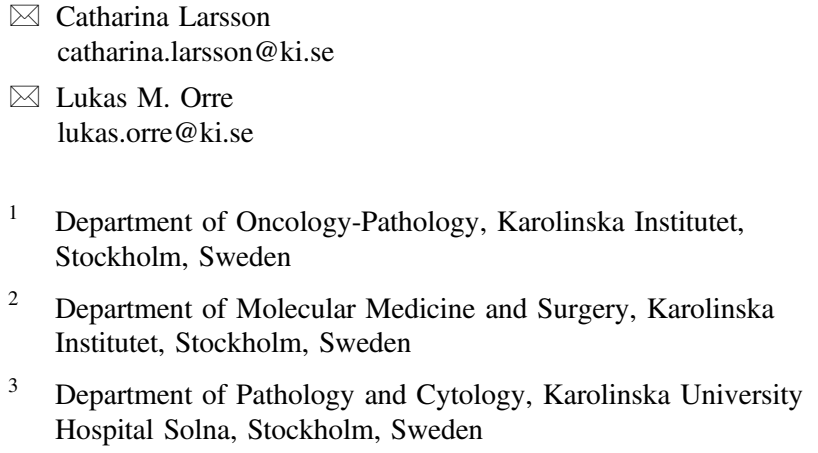

2 Department of Molecular Medicine and Surgery, Karolinska Institutet, Stockholm, Sweden

3 Department of Pathology and Cytology, Karolinska University Hospital Solna, Stockholm, Sweden

Supplementary information The online version of this article (https:// doi.org/10.1038/s41388-019-0938-8) contains supplementary material, which is available to authorized users.

\section{Introduction}

Small intestinal neuroendocrine tumor (SI-NET) is a rare disease with an incidence of around 1 per 100,000, but still the most common small intestinal malignancy [1]. SI-NETs develop from enterochromaffin cells, are positive for neuroendocrine markers, and the diagnosis is based on

4 SciLifeLab, Stockholm, Sweden

5 Department of Pharmacology, Cambridge University, Cambridge, UK

6 Department of Clinical Genetics, Linköping University Hospital, Linköping, Sweden

7 Uppsala Clinical Research Center, Uppsala University, Uppsala, Sweden

8 Department of Breast, Endocrine Tumours and Sarcoma, Karolinska University Hospital Solna, Stockholm, Sweden 
histopathological examination [2]. The patients typically present with one or more small primary tumors, often together with mesenteric lymph node metastases, and later development of distant metastases frequently to the liver. Although the tumors are commonly slow growing based on a low Ki-67 proliferation index, the clinical picture suggests that tumor cell disconnection and spreading occur early in the tumor development [3].

The primary treatment of SI-NET is surgical. In addition, the frequent expression of somatostatin receptors in SINETs has led to the development and successful application of somatostatin analog treatment. Although this treatment may improve symptoms and also in some patients delay tumor progression, additional therapeutic strategies need to be identified and developed for patients with metastatic disease.

SI-NETs are characterized by a few recurrent genetic aberrations such as copy number loss of chromosome 18, which is seen in $>70 \%$ of the cases [4]. The high frequency of this event spawned a search for putative tumor supressors located on chromosome 18 in SI-NET, resulting in the identification of DCC (deleted in colorectal cancer) as the so far best candidate [5]. Even though SI-NETs are in general mutationally quiet, the application of genome-wide sequencing identified $C D K N 1 B$ as a recurrently mutated gene in SI-NET with a frequency of $8 \%$ [6]. In addition, a broad molecular subtyping of SI-NET was recently reported where genomic, epigenomic, and transcriptomic profiles were used to suggest three molecular subtypes of SI-NET with differences in progression-free survival [7]. However, the molecular background of SI-NET development, specific oncogenic drivers, and mechanisms of metastatic spread still remain largely unknown. In addition, no study so far has integrated proteome-level information in the analysis of SI-NET from primary tumors.

$C D K N 1 B$ encodes p27, a bona-fide tumor suppressor that controls cell cycle entry through inhibition of cyclin/cyclin dependent kinase (CDK) activity. It has been thoroughly demonstrated that the CDK inhibitor p27 is regulated primarily by ubiquitination and subsequent proteasomal degradation [8], and that the ubiquitin E3-ligase responsible for the ubiquitination is the $\mathrm{SCF}^{\mathrm{Skp} 2}$ Cullin Ring Ligase (CRL) complex [9]. The $\mathrm{SCF}^{\mathrm{Skp} 2}$ complex was later shown to include Skp1, Cullin-1, Rbx1, and Skp2 as the core complex components [10]. Increased degradation of p27 in cancer has also been firmly established beginning with a seminal study where increased p27-specific proteolytic activity was coupled to low abundance of $\mathrm{p} 27$, which in turn was demonstrated as a powerful negative prognostic factor in colorectal cancer [11]. An overwhelming abundance of reports since then connects overexpression of Skp2 (the Fbox protein that recognize $\mathrm{p} 27$ ) with loss of $\mathrm{p} 27$ protein and poor prognosis in a long list of different cancer types [12].
Importantly, it has been shown that substrate recognition and activity of CRLs (such as $\mathrm{SCF}^{\mathrm{Skp} 2}$ ) is strongly dependent on neddylation of the Cullin subunit [13]. The neddylation process is similar to ubiquitination and includes E1, E2, and E3 enzymes that catalyze conjugation of target proteins with the ubiquitin-like protein NEDD8 (neural precursor cell expressed, developmentally downregulated 8) [14]. Cullin neddylation results in a conformational change of the CRL complex, eliminating the binding site for the CRL inhibitor Cand1 and, at the same time, providing a more open structure favoring polyubiquitination [15].

Here we performed in-depth proteomics profiling of 14 primary SI-NET tumors with focus on proteome-level differences between tumors from patients with and without liver metastasis. This clinical proteomics analysis implicated differences in the ubiquitination machinery, with higher expression of NEDD8 and RBX1 in tumors from patients with liver metastasis. To further investigate the importance of these findings, inhibition of neddylation was performed in SI-NET cell lines resulting in reduced proliferation and induction of apoptosis. Proteomics profiling of the effects of neddylation inhibition in two SI-NET cell lines resulted in identification of multiple upregulated proteins known to be substrates of CRLs including p27. Our findings thus suggest deregulation of CRLs as well as an alternative route for $C D K N 1 B / \mathrm{p} 27$ inactivation in SINET with possible connections to metastatic spread and therapy.

\section{Results}

\section{Proteomics profiling of primary SI-NETs indicate NEDD8 and RBX1 overexpression in patients with liver metastases}

In order to investigate the proteomic landscape of SI-NET, and especially to identify proteins connected to disease progression and metastatic spread, we performed in-depth proteomics profiling of 14 primary SI-NETs of which 7 presented with liver metastases, while 7 were without any detectable liver metastasis at the time of surgery (Supplementary Table S1). High-resolution isolectric focusing liquid chromatography-tandem mass spectrometry (HiRIEF-LC-MS)-based proteomics combined with relative quantification of proteins between samples (iTRAQ-labeling) resulted in the identification and quantification of 6775 proteins across all 14 SI-NET samples (Supplementary Table S2). Unsupervised analysis using principal component analysis of the 14 samples revealed no major batch effects between the 2 separate quantitative proteomics experiments ( 2 iTRAQ 8-plex kits), but indicated 2 potential outliers in the analysis (sample Screen-8 and -12 ; 
Supplementary Fig. S1a, b). Unsupervised Spearman's hierarchical clustering, however, did not indicate the two samples as outliers. Instead, this clustering resulted in a separation of samples based on experimental batch and, more interestingly, on liver metastases (Supplementary Fig. S1c).

Next, a supervised analysis was performed to identify protein-level differences between SI-NETs from patients with and without liver metastases. This analysis resulted in the identification of 538 differentially expressed proteins ( $p$ $<0.05$; Supplementary Table S3). Five proteins showed a more than twofold difference between groups including NEDD8, PSMB9, and TFF2 showing higher expression, and CADM3 and GAP43 showing lower expression in SINETs from patients with liver metastases (Fig. 1a). CADM3 has previously been proposed as a tumor suppressor in glioma where loss of CADM3 expression is frequently observed [16]; however, somatic CADM3 mutations and copy number aberrations seem rare in human cancers (COSMIC database). Also of potential importance, the closely related TFF2 family member TFF3 was recently shown expressed in SI-NETs, and in addition the serum level of TFF3 was associated with poor survival [17]. However, the previously shown connection between NEDD8 and cancer through its involvement in regulation of CRL activity [18] directed our focus and continued investigations to this protein.

To identify additional proteins with a potential functional coupling to NEDD8 in SI-NET, we performed a NEDD8 correlation analysis across the 14 samples. Strikingly, one of the most highly NEDD8 correlating proteins in this analysis was RBX1, another CRL core complex member (Fig. 1b and Supplementary Table S4). To further evaluate the expression of the 538 differentially expressed proteins across the 14 SI-NET samples, hierarchical clustering was performed, resulting in two main sample clusters (Fig. 1c). Six out of seven SI-NETs without liver metastases fell into the same cluster, whereas the other cluster included all tumors with liver metastases together with the remaining case without liver metastases (sample Screen-14).

The differential expression of NEDD8 and RBX1 in SINET together with the potential link to progression prompted us to investigate these genes further using public domain data generated in The Cancer Genome Atlas project (TCGA) [19]. Gene expression data covering 31 different cancer types (PanCancer dataset) indicated a significant spread in the expression of $N E D D 8$ both between and within cancer types (Fig. 1d). SI-NETs are not represented in the TCGA PanCancer dataset, but pheochromocytoma/ paraganglioma, the only neuroendocrine tumor type included, showed high expression of $N E D D 8$, supporting a role of this gene in NET. Further, a NEDD8 correlation analysis revealed that one of the genes with the highest NEDD8 correlation across the different cancer types was RBXI (Fig. $1 \mathrm{e}, \mathrm{f})$. These results indicate a co-regulation of $N E D D 8$ and $R B X 1$ at the transcriptional level, underscoring the functional association between these proteins and suggesting that some cancer types rely more on processes that are dependent on CRL activity for protein degradation.

In summary, our analysis shows differential NEDD8/ RBX1 expression at the protein level in SI-NETs, and a possible connection between CRL-dependent processes and progression of SI-NET.

\section{SI-NET cells are sensitive to the neddylation inhibitor pevonedistat}

Based on our proteomics analysis of SI-NETs we speculate that aberrant neddylation in SI-NETs is of possible importance for tumor progression and treatment strategies. To evaluate this hypothesis we used a neddylation inhibitor (pevonedistat, MLN4924) that targets the NEDD8activating enzyme 1 (NAE1) [20]. The most well-studied targets of neddylation are cullins, core proteins in CRLs, which require neddylation for activation, and therefore pevonedistat acts as an indirect inhibitor of CRL activity.

To study the effects of neddylation inhibition, four different NET cell lines (CNDT2 and HC45, both originating from liver metastasis of SI-NET; BON-1, pancreatic origin; NCI-H727, bronchial origin) were treated with pevonedistat at seven different concentrations ranging from 100 to $2000 \mathrm{nM}$. After 3 days of treatment, decreased proliferation, as assessed by Bromodeoxyuridine (BrdU) incorporation, was observed in all four cell lines (Fig. 2a and Supplementary Fig. S2), with the most dramatic effect observed in the SI-NET cell lines CNDT2 and HC45 (IC $50: 149 \mathrm{nM}$ and $322 \mathrm{nM}$, respectively, Fig. 2a). Previous studies in other cancer types have shown pevonedistat $\mathrm{IC}_{50}$ values in the range of $50-1000 \mathrm{nM}$ [20], indicating that at least CNDT2 and $\mathrm{HC} 45$ are sensitive to pevonedistat treatment. To further evaluate the sensitivity of NET cells in relation to other cancer cells, we used data generated in the Genomics of Drug Sensitivity in Cancer (GDSC) project [21]. The GDSC data contain pevonedistat response measurements for 692 different cancer cell lines with a median pevonedistat $\mathrm{IC}_{50}$ value of $1100 \mathrm{nM}$ (Fig. 2b). Both SI-NET cell lines investigated here showed an $\mathrm{IC}_{50}$ value well below the GDSC median, and in particular CNDT2 cells were comparable to the more sensitive cell lines in the GDSC analysis. Grouping the pevonedistat sensitivity measurements from GDSC by cell origin indicated large differences in sensitivity within each tissue type (Fig. 2c). To identify potential pevonedistat response markers, mRNA profiling data were downloaded from GDSC and used to perform a differential expression analysis between the pevonedistat-sensitive and -resistant GDSC cell lines as indicated in Fig. 2b. This 
a

\section{Liver metastasis vs no liver metastasis} Differential Analysis $n=6775$

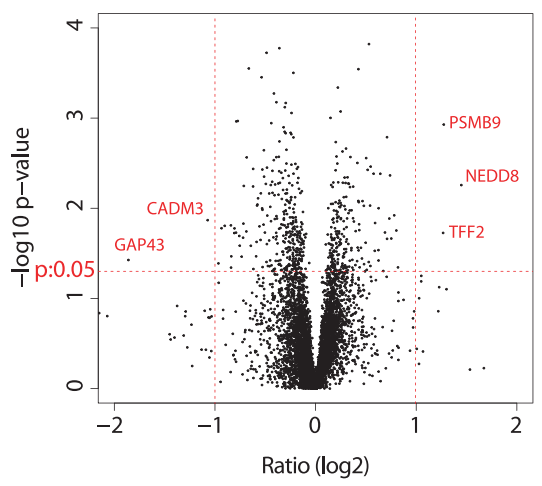

b

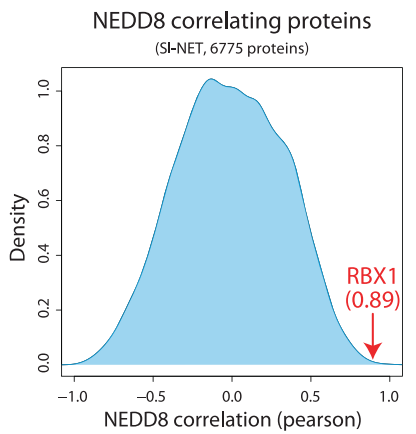

C

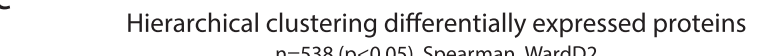
$\mathrm{n}=538(\mathrm{p}<0.05)$, Spearman, WardD2

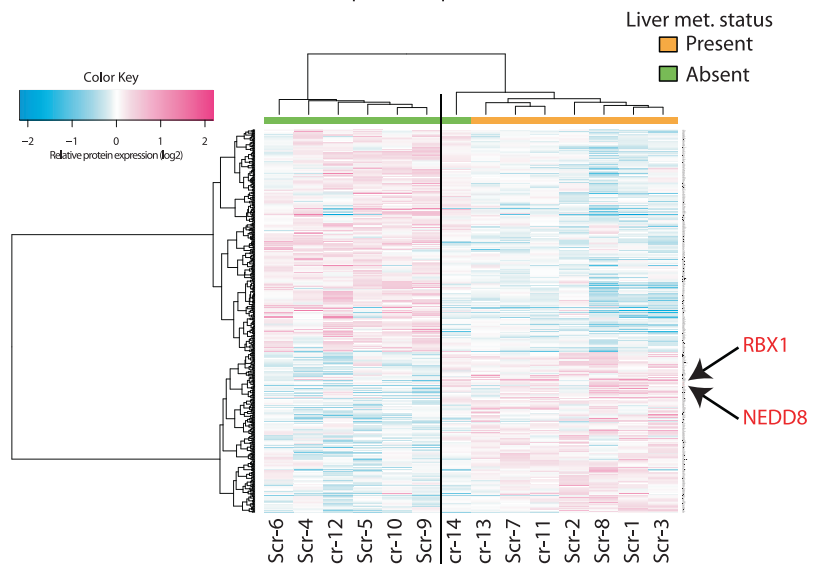

NEDD8 expression mRNA (TCGA PanCancer, 9755 samples)
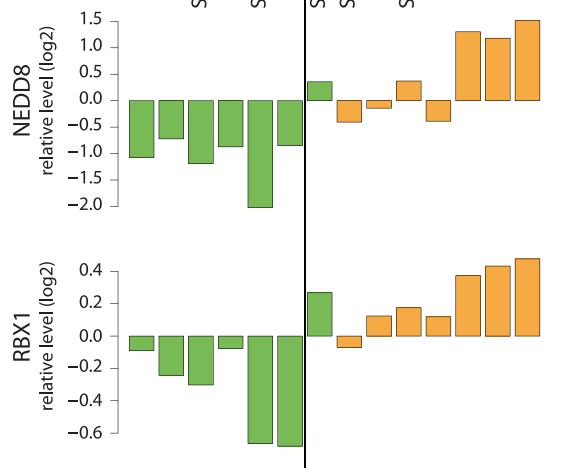

d

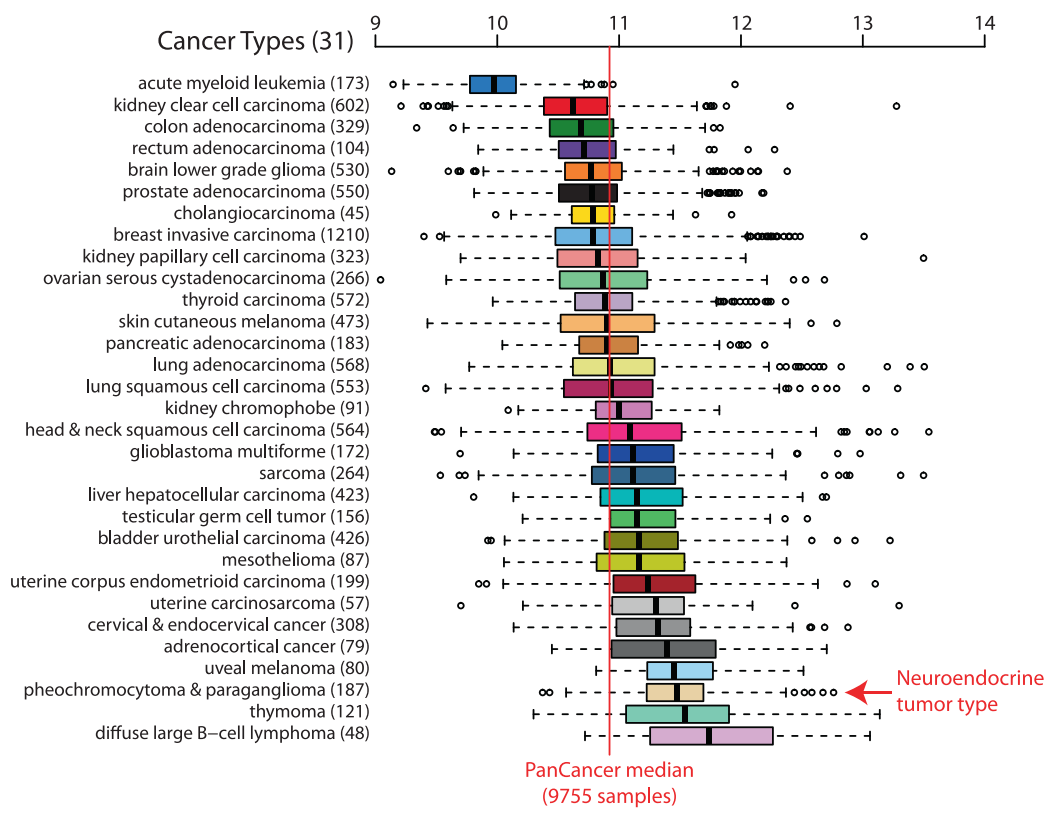

e NEDD8 correlating mRNA

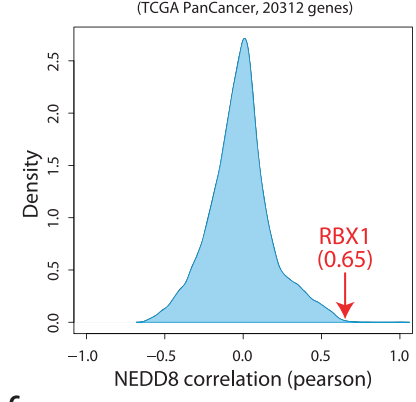

$f$

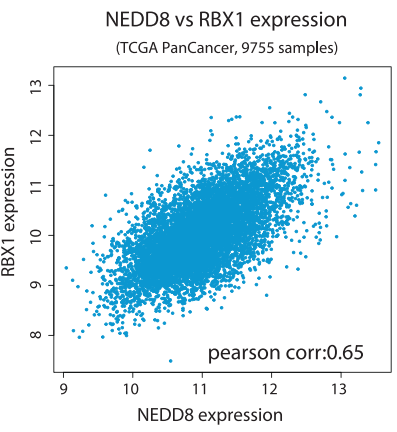

analysis indicated that none of the neddylation machinery components (NAE1, UBA3, UBE2M, RBX1, SENP8) or the cullins were useful as predictive mRNA level biomarkers for pevonedistat response (Fig. 2d, NEDD8 itself was not part of the GDSC data). Instead, the analysis indicated that epithelial markers (EPCAM, ESRP1, CDH1, and KRT19) were significantly lower in pevonedistat-sensitive cell lines, whereas the mesenchymal marker VIM was higher. The 
Fig. 1 Proteomics profiling of SI-NET. a Volcano plot of the results from differential analysis of protein levels between tumors from patients with/without liver metastasis. Indicated in the plot are proteins with $p$-value below 0.05 and more than twofold different expression between groups. b Density plot showing the distribution of NEDD8 correlations for 6775 proteins quantified in the proteomics analysis. Indicated in red is RBX1, a core complex member of Cullin-RING ubiquitin ligases. $\mathbf{c}$ Clustering of differentially expressed proteins $(p<$ 0.05 ) between cases with and without liver metastases. The bar chart below shows relative protein expression levels from mass spectrometry analysis for NEDD8 and RBX1 in individual SI-NETs. The vertical line indicates the division of samples into two clusters. d NEDD 8 mRNA expression across 31 cancer types in the TCGA PanCancer dataset. Indicated in the boxplot is pheochromocytoma and paraganglioma, a neuroendocrine tumor (NET) type. e Density plot showing the distribution of $N E D D 8$ correlations at mRNA level for 20312 transcripts quantified in the TCGA project. Indicated in red is $R B X 1$. f Scatterplot showing the expression of $N E D D 8$ and $R B X 1$ in all 9755 samples in the TCGA dataset

indication that pevonedistat sensitivity is higher in nonepithelial cells would support a potential use of pevonedistat in NET cells as indicated by the relatively low EPCAM expression in pheochromocytoma/paraganglioma in the TCGA PanCancer dataset (Fig. 2e). It should be noted although that pheochromocytoma/paraganglioma is an imperfect proxy for SI-NETs in this case, as SI-NETs show varying expression of epithelial markers [22].

\section{Pevonedistat treatment of SI-NET cells results in stabilization of multiple CRL substrates with tumor suppressor function including p27}

To investigate the specific activity of pevonedistat in SINET cells, we used proteomics profiling of pevonedistattreated cells to identify stabilized proteins. Based on the results from proliferation analyses, CNDT2 and HC45 (both of SI-NET origin) were selected for further analysis. To identify protein-level regulation in response to pevonedistat treatment, CNDT2 and HC45 cells were analyzed by proteomics in biological duplicates as untreated or after treatment with pevonedistat $(400 \mathrm{nM})$ for $3,6,12$, and $24 \mathrm{~h}$. HiRIEF-LC-MS proteomics profiling with relative quantification using TMT isobaric labeling resulted in the identification and quantification of 10,551 and 11,298 proteins across all samples in CNDT2 and HC45 experiments, respectively (Supplementary Tables S5 and S6).

As only duplicate samples were analyzed for each time point, differential expression analysis was performed based on a fold change cutoff defined by the quantitative robustness in each cell line separately (Supplementary Fig. S3). Proteins with consistent up- or downregulation in the two replicates were included in the further analyses. Increasing numbers of regulated proteins over time were observed, with more proteins being upregulated than downregulated in both cell lines (Fig. 3a). In addition, the response appeared to be more rapid in CNDT2 cells, which is also supported by the higher sensitivity to pevonedistat treatment as discussed above. Our primary interest in the proteomics analysis of pevonedistat effects was to identify stabilized proteins, including potential CRL targets in SI-NET cells. In total, 651 proteins were upregulated $24 \mathrm{~h}$ after treatment with 366 proteins upregulated in CNDT2 and 373 proteins in $\mathrm{HC} 45$, of which 88 proteins were common for both cell lines (Fig. 3b and Supplementary Table S7). Interestingly, a closer analysis of these 88 proteins indicated that most of them were stabilized already $3 \mathrm{~h}$ after treatment (Fig. 3c). Such early effects would indicate posttranscriptional regulation such as altered degradation, as secondary effects that rely on transcription would have little time to manifest themselves at the protein level already $3 \mathrm{~h}$ after treatment. Indeed, a comparison with previously published lists of candidate CRL substrates [23] revealed a large number of proteins in common (Fig. 3d), many of which are wellknown substrates of CRLs, as exemplified by NFE2L2/Nrf2 and $C D K N 1 B / \mathrm{p} 27$.

With the hypothesis that CRL overactivation can contribute to the development of cancer through degradation of tumor suppressor proteins, we investigated whether some of the stabilized proteins here identified had a previously known connection to cancer. As part of the catalog of somatic mutations in cancer (COSMIC), the Cancer Gene Census is a collection of genes that have been causally implicated in cancer [24]. The latest version (downloaded 25 October 2018) contains 719 genes, and out of these 719 genes, 43 were identified at the protein level as stabilized after treatment with pevonedistat in SI-NET cells (Fig. 3e). Out of these, 22 were associated with a tumor suppressor role in cancer, including 3 proteins that were stabilized in both CNDT2 and HC45 cell lines in response to pevonedistat treatment (CDKN1B/p27, NFE2L2/Nrf2, and SPOP).

Further, 168 of the 651 proteins that were stabilized in response to pevonedistat were also identified and quantified in the SI-NET clinical proteomics experiment. For these proteins, a NEDD8 correlation analysis was performed to investigate potential functional association in clinical samples (Fig. 3f). Interestingly, one of the highest correlating proteins was p27 (Pearson's corr: 0.57), supporting a potential functional link between NEDD8 and p27 in clinical samples.

True substrates regulated directly by CRL-dependent ubiquitination and proteasomal degradation should be stabilized also by inhibition of the proteasome. To further narrow the list of candidates, we therefore performed an additional MS-based proteomics experiment where we treated CNDT2 cells with the protesome inhibitor bortezomib, alone or in combination with pevonedistat. This experiment resulted in the identification and quantification of 9434 proteins, with an overlap of 8507 proteins to the 
a

Pevonedistat response in CNDT2

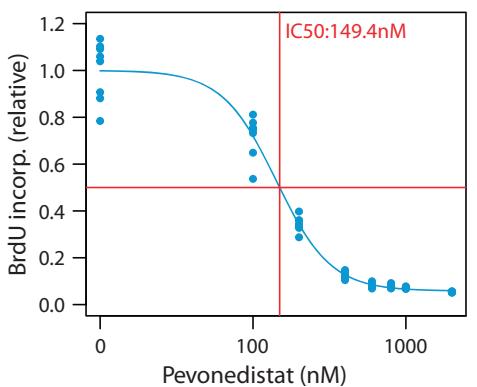

Pevonedistat response in $\mathrm{HC} 45$

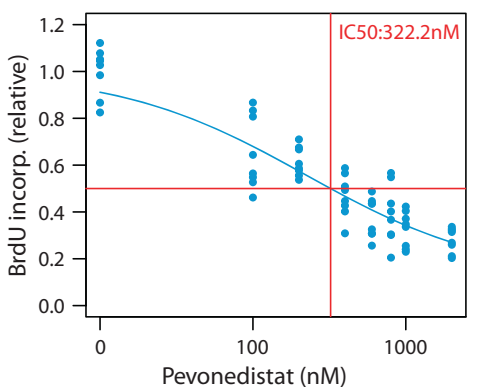

d

DE analysis pevonedistat Sensitive vs Resistant cell lines (mRNA data from GDSC)

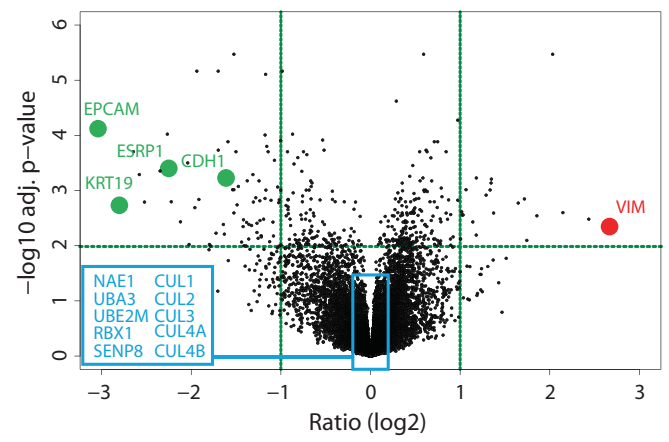

b

Pevonedistat response in GDSC 692 Cell Lines

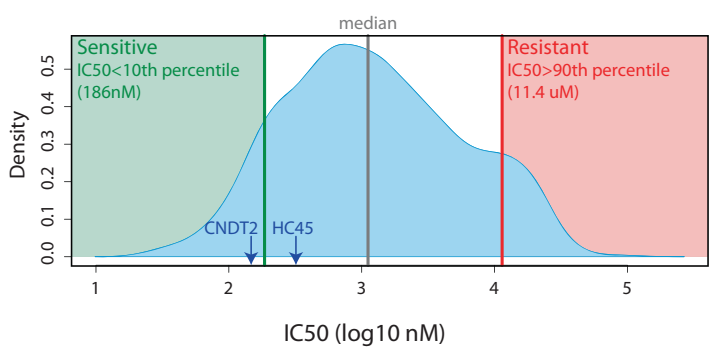

C

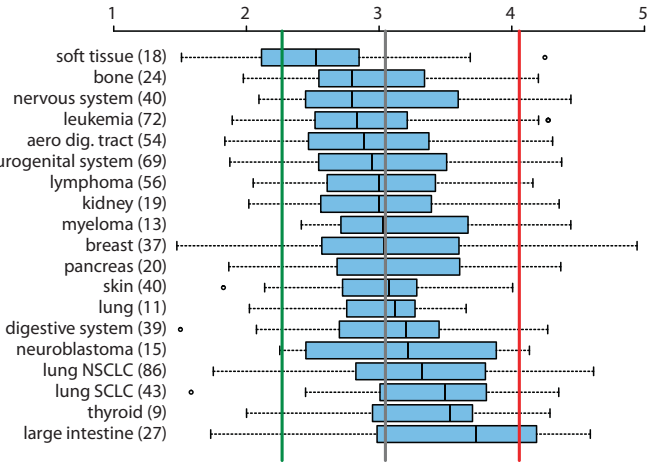

e

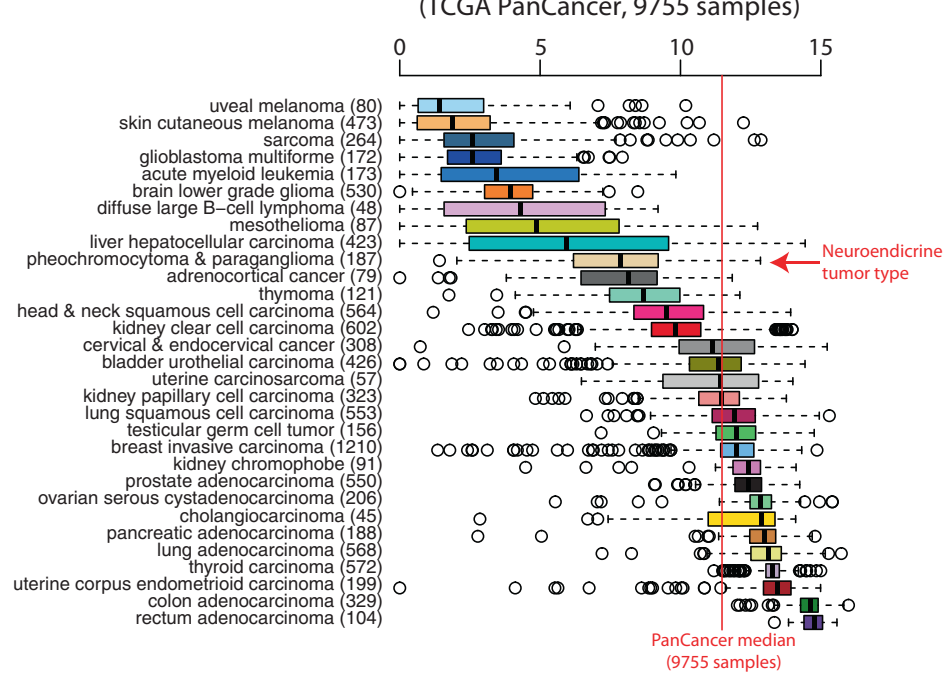

Fig. 2 SI-NET cells are sensitive to pevonedistat. a Drug sensitivity analysis by BrdU incorporation in SI-NET cell lines after pevonedistat treatment to inhibit neddylation. CNDT2 (top) or HC45 (bottom) cells were treated with pevonedistat for $72 \mathrm{~h}$ at different concentrations before the assay readout. b Density plot showing the distribution of pevonedistat $\mathrm{IC}_{50}$ values across 692 different cell lines as reported in the GDSC project. Indicated in the plot are the 10th and 90th percentiles used to define cell lines as sensitive or resistant. Also indicated in the plot are the measured $\mathrm{IC}_{50}$ values for the two SI-NET cell lines. c Boxplot showing the distribution of pevonedistat $\mathrm{IC}_{50}$ values per

data generated after pevonedistat treatment alone in CNDT2 cells as described above (Fig. 4a and Supplementary Table S8). In total, bortezomib treatment of CNDT2 cells resulted tissue of origin for the cell lines in the GDSC panel. d Vulcano plot showing the output from a differential expression (DE) analysis between pevonedistat-sensitive and -resistant cell lines based on GDSC mRNA data. Indicated in the plot are markers of epithelial (green) or mesenchymal (red) lineage. Indicated in blue are genes with known role in CRL-dependent processes. e Epithelial marker EPCAM mRNA expression across 31 cancer types in the TCGA PanCancer dataset. Indicated in the boxplot is pheochromocytoma and paraganglioma, a neuroendocrine tumor type

in stabilization of 629 proteins $(1.5$-fold, adjusted $p$-value $<$ 0.01 ) out of which 84 were found stabilized also by the pevonedistat treatment alone, with 38 also stabilized in the 
a
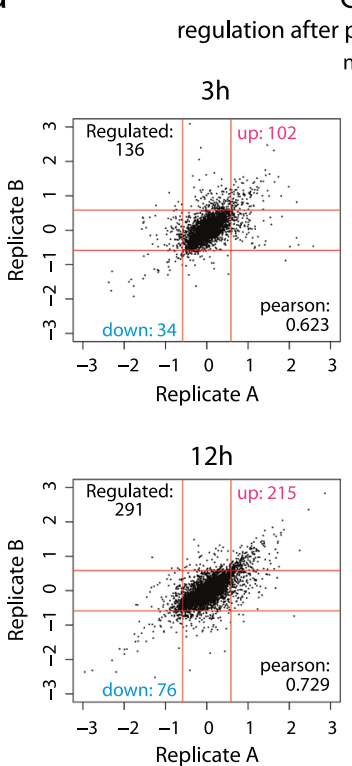

b

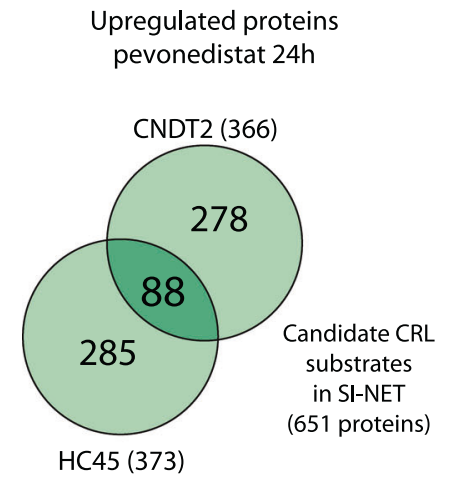

d

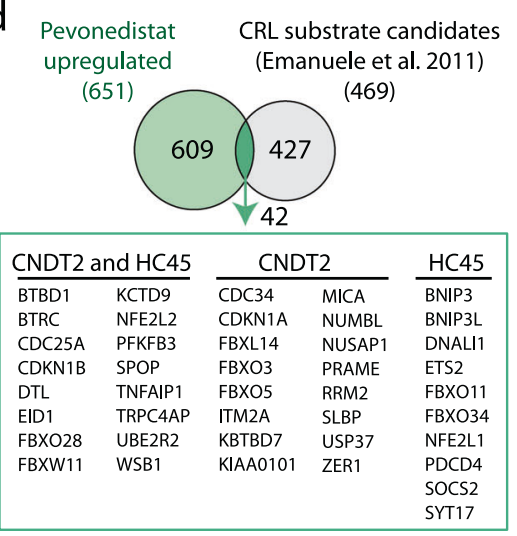

CNDT2

pevonedistat treatment

$n=10551$
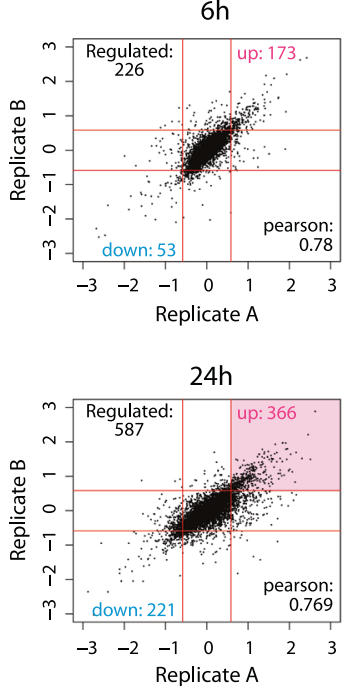

$\mathrm{HC} 45$

regulation after pevonedistat treatment

$n=11298$
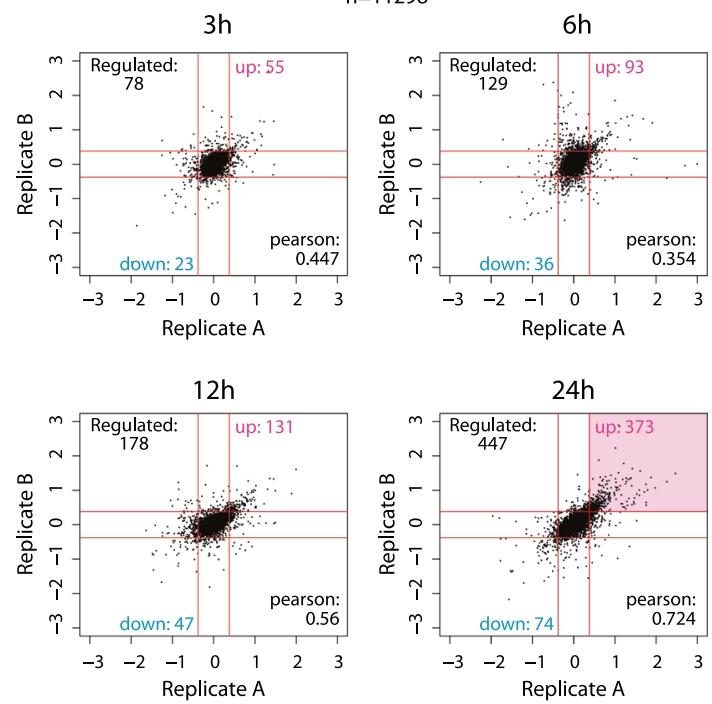

C

Proteins upregulated in both CNDT2 and HC45

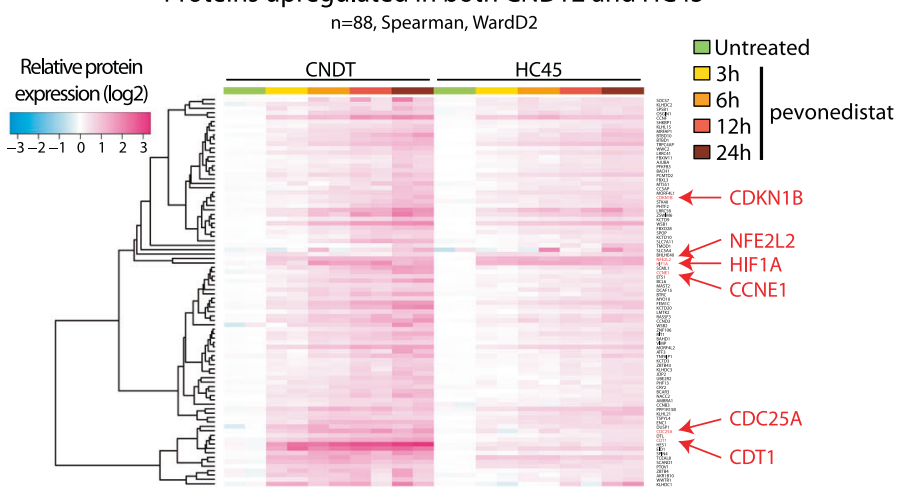

e

Pevonedistat
upregulated

$$
\text { (651) }
$$

651)

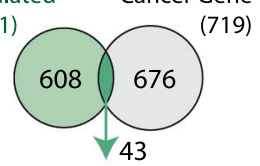

\begin{tabular}{|llll|}
\hline CDKN1B & FBXO11 & CCNE1 & STIL \\
SPOP & MLF1 & HIF1A & CARD11 \\
AXIN1 & NRG1 & BCL6 & JUN \\
BRIP1 & CASP3 & CCND3 & CCND1 \\
BUB1B & PTPRD & WWTR1 & ETV4 \\
KDM5C & NFE2L2 & FOXA1 & MYC \\
SMARCD1 & CDKN1A & ABL2 & TFE3 \\
TRAF7 & RUNX1 & CCND2 & RGPD3 \\
CASC5 & TRIM24 & CTNNB1 & SDC4 \\
CBFA2T3 & BTK & ETV5 & SRGAP3 \\
PER1 & TP63 & SIX2 & \\
\hline
\end{tabular}

TSG TSG/Oncogene Oncogene

In bold text are proteins stabilized in both CNDT2 and HC45 $f$

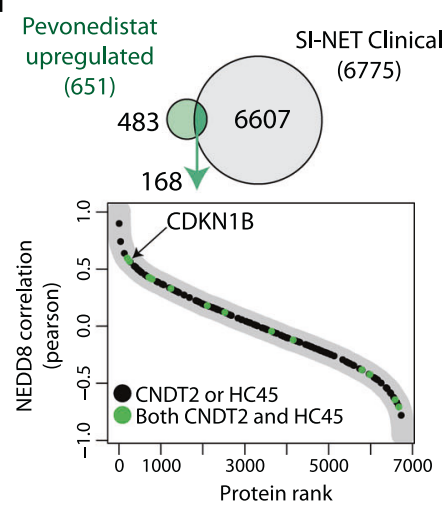

HC45 cells (Fig. 4a, b). Further, our analysis showed that when combining bortezomib and pevonedistat, a subset of proteins stabilized by bortezomib treatment alone was even more stabilized by the combination treatment, strongly indicating that these proteins are substrates of CRL- dependent ubiquitination and proteasomal degradation (Fig. 4c). Out of these proteins, 30 (including p27; Fig. 4d) were identified as candidate CRL substrates by pevonedistat treatment alone in CNDT2 cells, including 21 proteins also stabilized in HC45 cells after pevonedistat treatment 
Fig. 3 Proteomics profiling of response to pevonedistat in SI-NET cell lines. a Scatterplots showing the results from the quantitative proteomics experiments of pevonedistat in CNDT2 cells (left) and HC45 cells (right) at four different time points each. Plotted values represent $\log 2$ ratio between the indicated sample and average of untreated duplicate samples. Red lines indicate the cutoffs for regulation as defined in Supplementary Fig. 2. In each scatterplot, the number of proteins regulated in both cell lines are indicated as well as the overall correlation between replicate A and B. b Venn diagram showing the overlap between CNDT2 and HC45 in proteins upregulated $24 \mathrm{~h}$ after pevonedistat treatment. c Heatmap showing proteins detected as upregulated at $24 \mathrm{~h}$ in both CNDT2 and HC45. Indicated in the heatmap are examples of CRL substrates that are stabilized in response to pevonedistat treatment. d Overlap analysis between proteins identified as stabilized after pevonedistat treatment in SI-NET cells and previously identified CRL substrate candidates. e Overlap analysis between pevonedistat-stabilized proteins in SI-NET cells and in oncogenes/tumor suppressor genes (TSG) according to COSMIC Cancer Gene Census. f Overlap analysis between pevonedistatstabilized proteins in SI-NET and proteins quantified in SI-NET clinical samples (top). Bottom plot shows a correlation analysis in clinical samples between pevonedistat-stabilized proteins and NEDD8 based on clinical proteomics data. The x-axis indicate the rank of stabilized proteins in relation to all proteins the SI-NET clinical dataset $(n=6775)$ with respect to the NEDD8 correlation

(Fig. 4e). Several of these proteins are prototype CRL substrates and four of the proteins including p27 (CDKN1B), p21 (CDKN1A), nrf2 (NFE2L2), and SPOP are tumor suppressors according to COSMIC.

In summary, our analysis shows that treatment of SINET cells with the neddylation inhibitor pevonedistat results in a rapid stabilization of CRL targets, including well-known tumor suppressors such as p27.

\section{Pevonedistat treatment of NET cells results in increased p27 level and induction of apoptosis}

To further investigate the effects of pevonedistat treatment in NET cells, CNDT2, HC45, BON-1, and NCI-H727 cells were treated for $72 \mathrm{~h}$ with pevonedistat in increasing concentrations from 100 to $1600 \mathrm{nM}$. Western blotting analysis of protein neddylation using anti-NEDD8 antibody revealed the reduction of a band ( $~ 90 \mathrm{kDa})$ corresponding to the predicted size of Cullin conjugated with NEDD8 in all cell lines (Fig. 5a). The expression level of unconjugated NEDD8 at $9 \mathrm{kDa}$ as well as unconjugated Cullin-1 did not demonstrate a difference between untreated and treated cells at different concentrations. This result is in line with previous literature showing inhibition of Cullin neddylation as an important effect of pevonedistat treatment [20]. Further, western blotting analysis of p27 confirmed the results from the proteomics analysis as increased level of p27 was observed in all cell lines in a dose-dependent manner.

The BrdU incorporation assay clearly showed that treatment with pevonedistat results in reduced proliferation (Fig. 2a and Supplementary Fig. S2). To evaluate induction of apoptosis in response to pevonedistat, poly(ADP-ribose) polymerase (PARP) cleavage was assayed using Western blot. In three out of four NET cell lines, a dose-dependent increase in cleaved PARP (cPARP) was detected (Fig. 5a). To further confirm the induction of apoptosis in response to pevonedistat treatment in NET cells, Annexin V staining was assessed by flow cytometry. After 3 days of pevonedistat treatment, the proportion of cells that were stained by Annexin $\mathrm{V}$ was increased in all cell lines, indicating the induction of apoptosis (Fig. 5b). Collectively, these results show that inhibition of neddylation using pevonedistat treatment results in a dose-dependent stabilization of p27 as well as induction of apoptosis in all NET cell lines examined.

\section{p27 stability is dependent on NEDD8 and RBX1 levels}

According to our hypothesis, altered levels of NEDD8 and RBX1 could contribute to altered CRL activity and consequently also to altered stability of CRL substrates such as p27. To investigate this further, we used small interfering RNA (siRNA)-based silencing to monitor the effects of knocking down NEDD8 and RBX1 in CNDT2 cells. Importantly, silencing of either NEDD8 (Fig. 6a) or RBX1 (Fig. 6b) resulted in a clear increase in $\mathrm{p} 27$ protein levels, strongly indicating that either one of these proteins are rate limiting for the CRL activity and the degradation of CRL substrates.

As shown above, many different substrates are affected by CRL inhibition using pevonedistat in SI-NET cells. To investigate the relative contribution of p27 to the effects of pevonedistat, we used siRNA-based silencing of p27 in a rescue experiment. p27 is efficiently degraded via the proteasome and, therefore, knockdown efficiency was evaluated in pevonedistat-treated CNDT2 cells (Fig. 6c). As a readout of pevonedistat effect, Annexin V staining was assessed by flow cytometry to evaluate early apoptotic events $24 \mathrm{~h}$ after treatment (Fig. 6c, Supplementary Fig S4, and Supplementary Table S9). This experiment showed that silencing of p27 using two different siRNAs resulted in a partial rescue of pevonedistat effects as indicated by a decrease in early apoptotic cells (AnnV+/PI-) and an increase in healthy cells (AnnV-/PI-).

Further, similar to what was seen after inhibition of CRL activity using pevonedistat, silencing of NEDD8 and RBX1 resulted in induction of apoptosis as indicated by increased PARP cleavage (Fig. 6d). Part of this effect was dependent on p27, as we noted a clear reduction of PARP cleavage after silencing of $\mathrm{p} 27$.

In conclusion, these experiments show a causal link between cellular NEDD8 or RBX1 levels and p27 levels, and that the cellular effects of CRL inhibition using pevonedistat or NEDD8/RBX1 silencing is in part dependent on stabilization of $\mathrm{p} 27$. 


\section{CNDT2 proteomics experiments ID overlap}
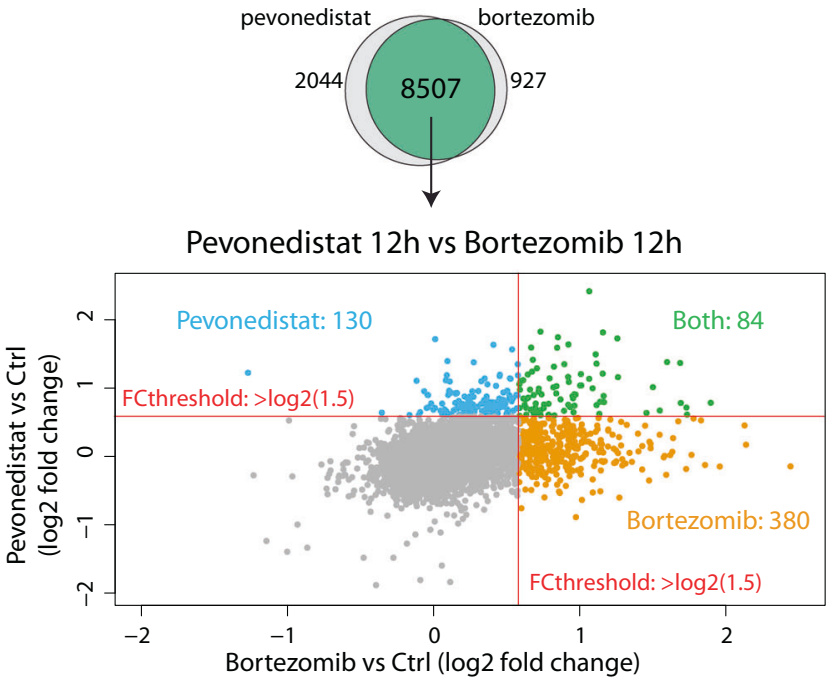

C

\section{Bortezomib vs bortezomib+pevonedistat combination in CNDT2 cells}

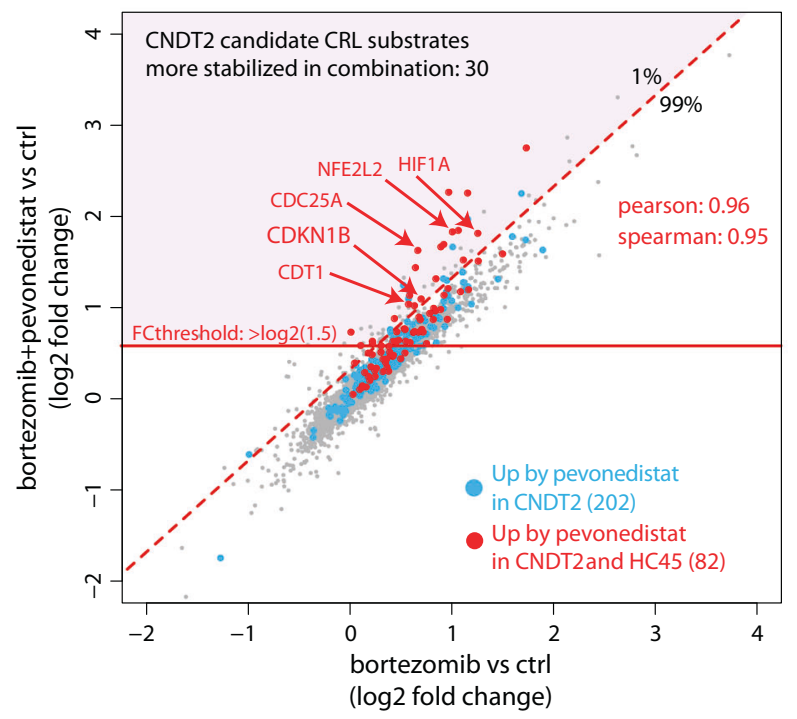

Fig. 4 CRL substrate evaluation by proteomics profiling of bortezomib effects in CNDT2 SI-NET cells. a Venn diagram showing identification overlap in CNDT2 cells between proteomics profiling of pevonedistat effects (as described in Fig. 3) and bortezomib effects (top), and scatterplot indicating proteins found to be stabilized by monotherapy with pevonedistat and/or bortezomib (bottom). b Vulcano plot showing protein-level regulation in response to bortezomib treatment of CNDT2 cells. Indicated in the plot are proteins also found to be stabilized by pevonedistat treatment in CNDT2 cells (blue) or both CNDT2 cells and HC45 cells (red) as described in Fig. 3. c Scatterplot showing CNDT2 cells' protein-level regulation in response to bortezomib treatment alone or in combination with pevonedistat. Dashed line indicate 99th percentile of the comparison between effects after b

Regulation after bortezomib treatment in CNDT2 cells $\mathrm{n}=9434$ proteins

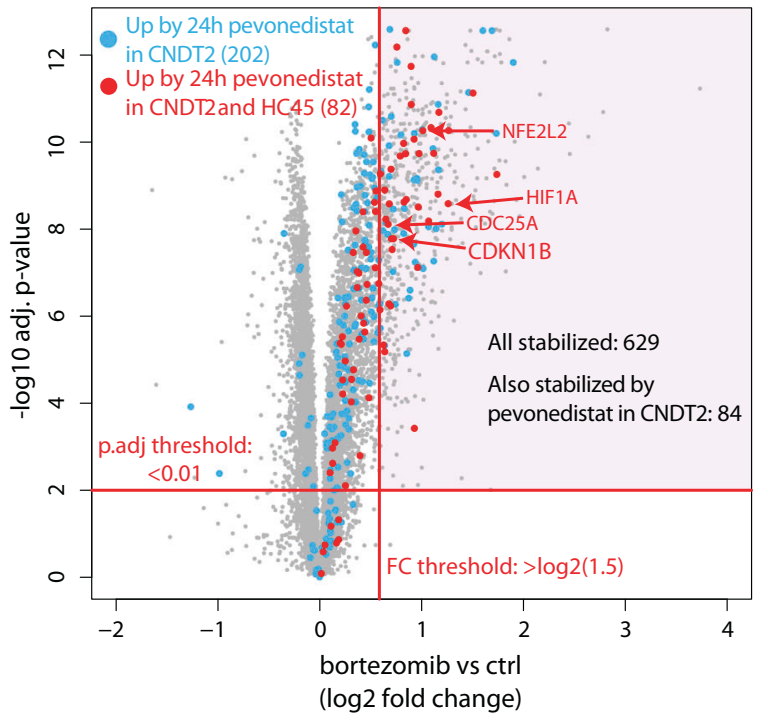

d
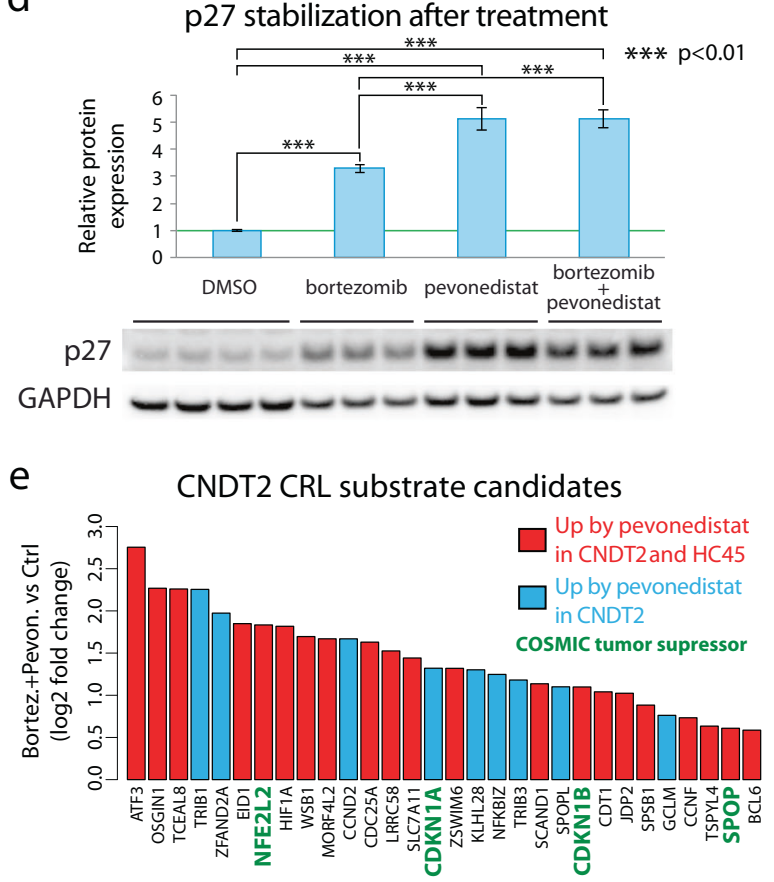

treatment with bortezomib/pevonedistat combination and bortezomib alone. Pink area indicate proteins that are stabilized to a higher degree after combination treatment. d Western blotting showing p27 levels in response to treatment of CNDT2 cells with bortezomib and pevonedistat alone or in combination. The barplot indicates relative protein levels normalized to GAPDH. Western blotting quantification was based on three independent experiments with barplot error bars indicating SD and $p$-values calculated by Student's $t$-test. e Barplot showing the 30 SI-NET CRL substrate candidates that were stabilized more in response to bortezomib pevonedistat combination than bortezomib alone in CNDT2 cells. Indicated in green are proteins defined as tumor suppressors (TSG) in COSMIC 
a

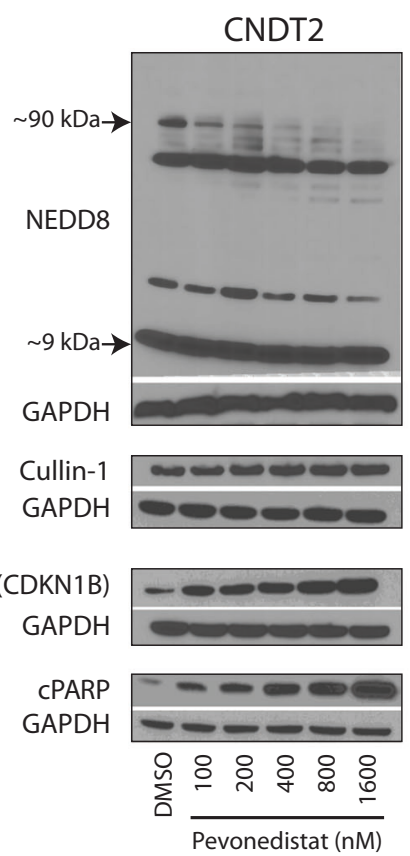

$\mathrm{HC} 45$
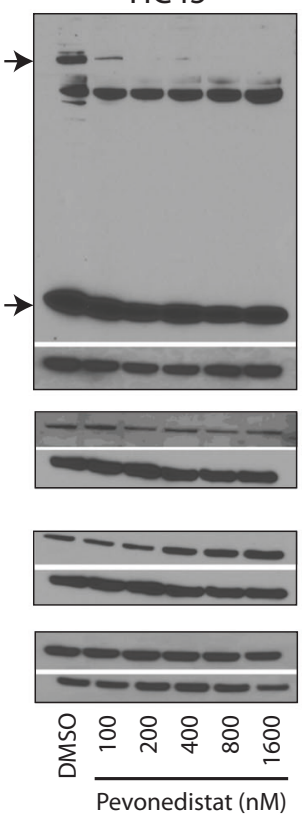

BON-1
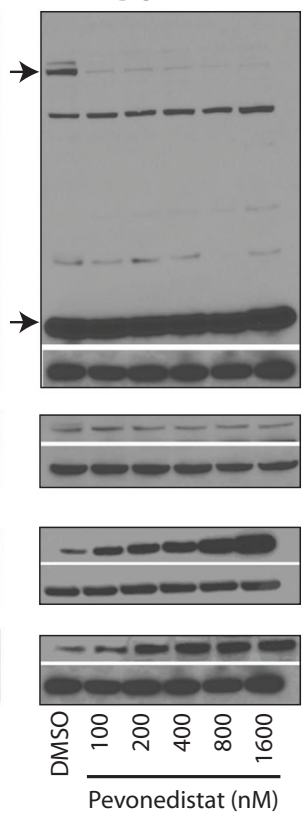

$\mathrm{NCl}-\mathrm{H} 727$
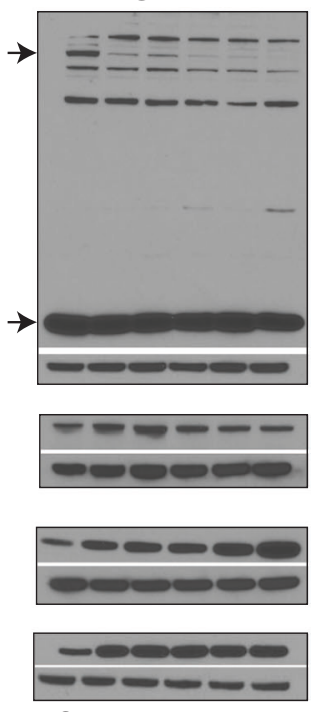

商 $\frac{\circ}{\text { Pevonedistat }(\mathrm{nM})}$

b

Pevonedistat
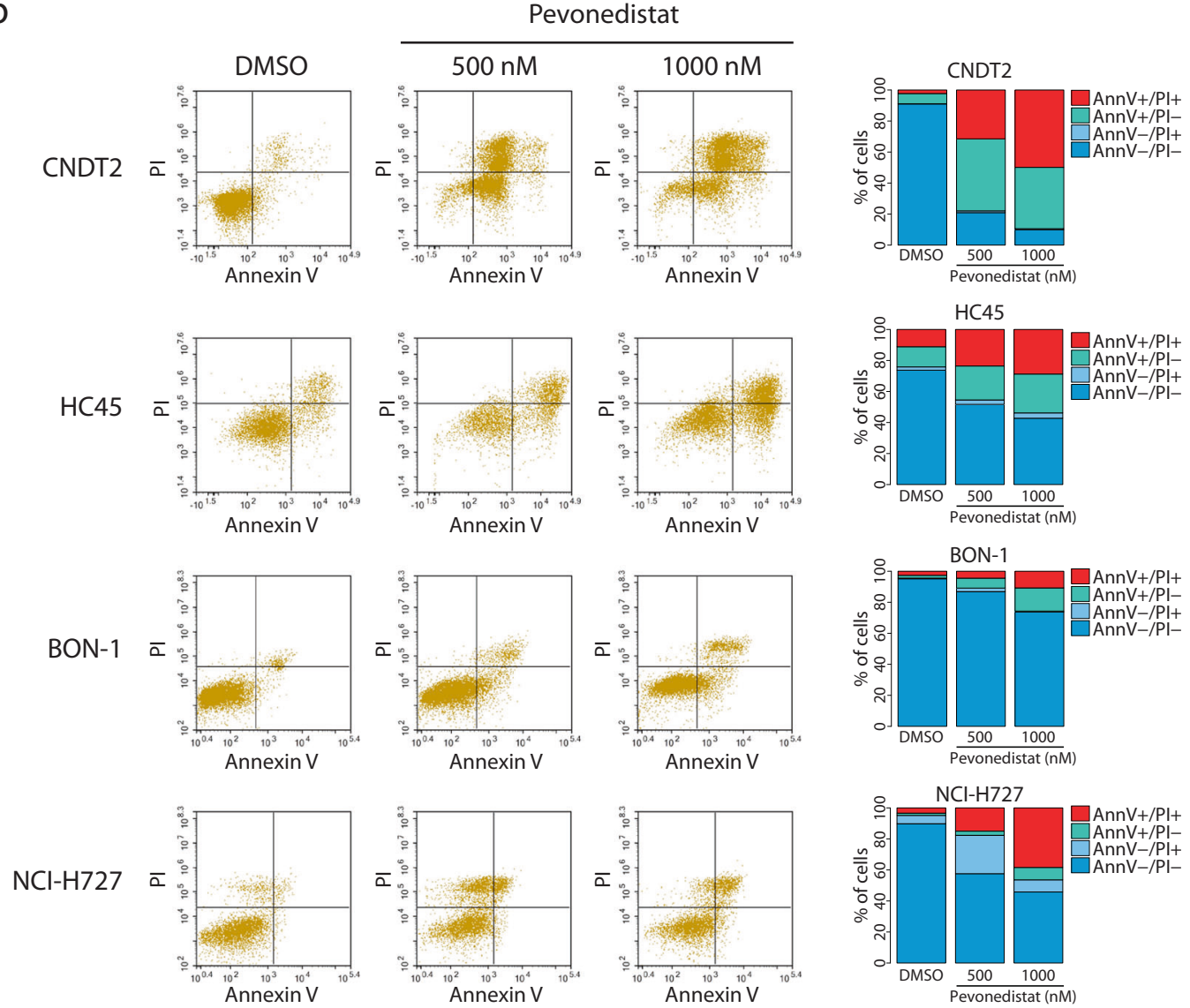

Fig. 5 Pevonedistat treatment results in stabilization of p27 and induction of apoptosis in SI-NET cells. a Western blotting analysis of p27, cleaved PARP (cPARP), Cullin-1, and NEDD8 in NET cell lines after pevonedistat treatment at different concentrations for $72 \mathrm{~h}$. Indicated by arrows in the NEDD8 blot are bands corresponding to neddylated cullin $(\sim 90 \mathrm{kDa})$ as well as unconjugated NEDD8 $(\sim 9 \mathrm{kDa})$.

For all blots, GAPDH was used as loading control. b Apoptosis assay $72 \mathrm{~h}$ after pevonedistat treatment in NET cell lines. Representative propidium iodide (PI) and Annexin V-FITC flow cytometry plots are shown for DMSO-treated control cells and cells treated with 500 and $1000 \mathrm{nM}$ pevonedistat. At the right, the proportion of cells positive for propidium iodide (PI) and Annexin V-FITC (AnnV) is shown $(n: 3)$ 
NEDD8 silencing results in increased p27

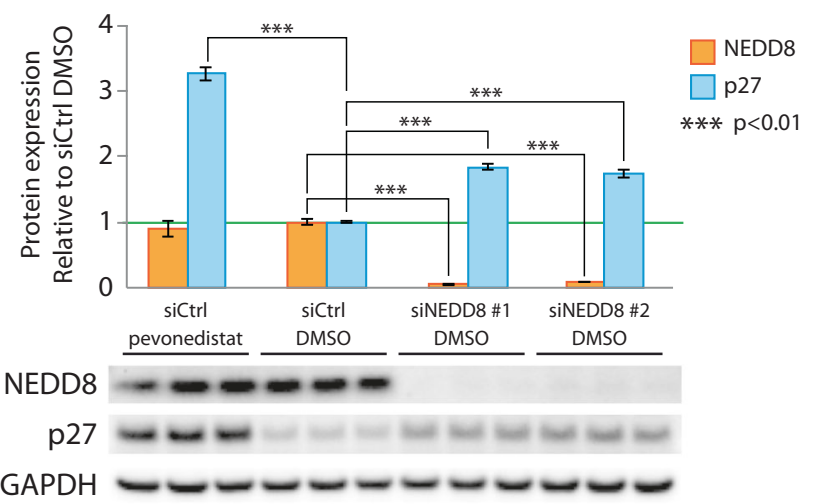

b

RBX1 silencing results in increased $p 27$

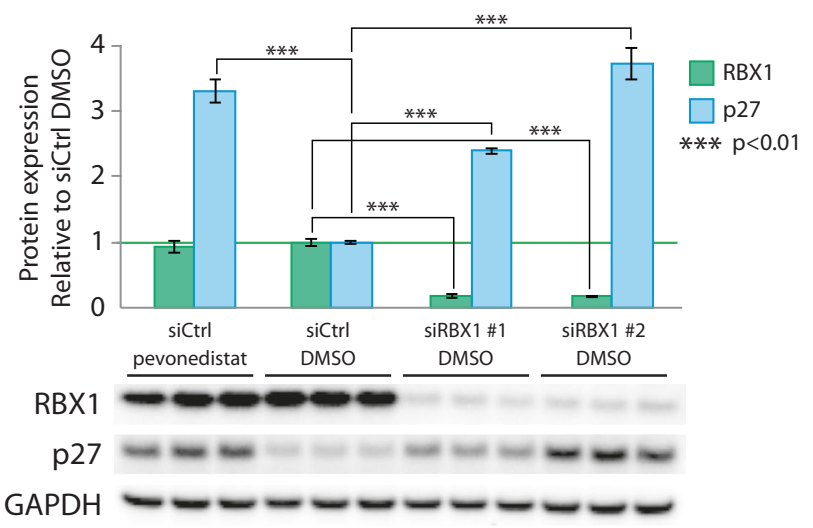

C p27 silencing results in reduced apoptosis after pevonedistat treatment
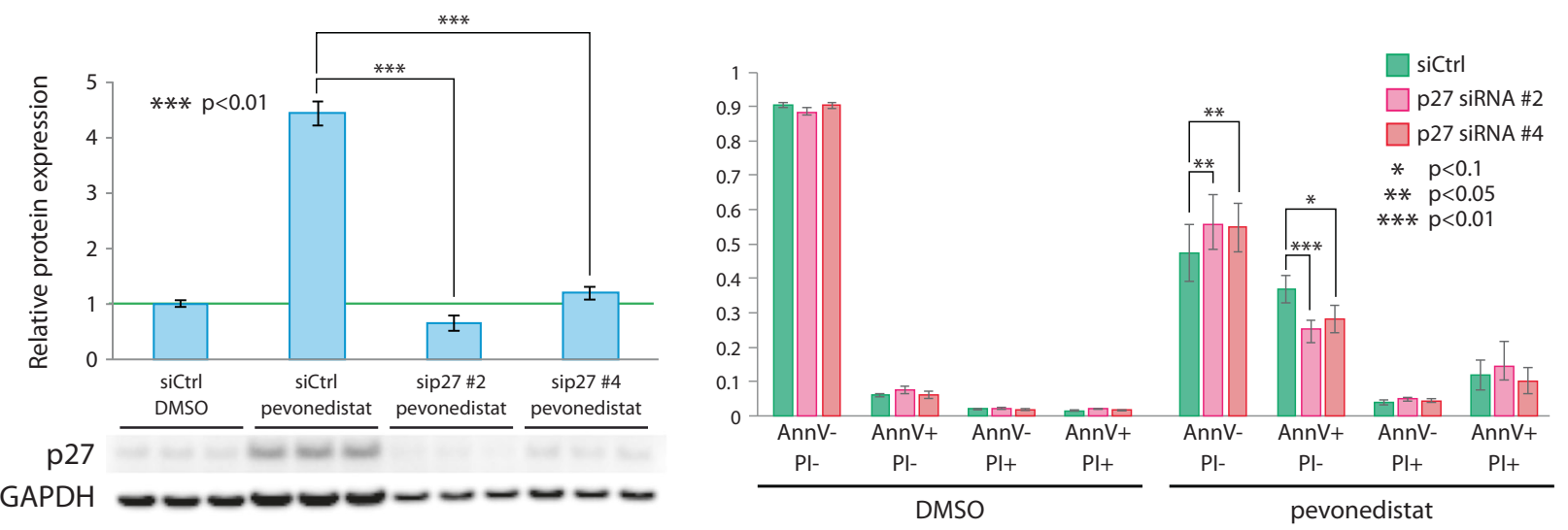

d

NEDD8 and RBX 1 silencing results in PARP cleavage that is reduced by p27 silencing
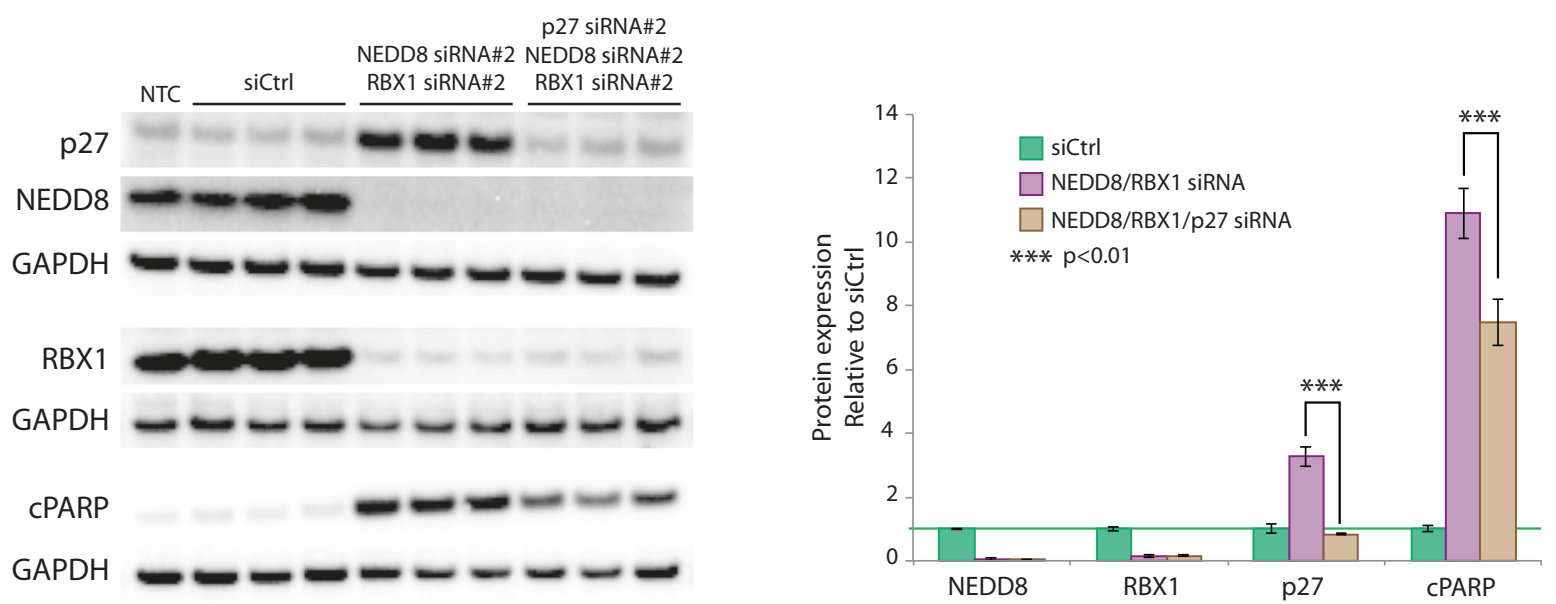

\section{Clinical evaluation of CDKN1B/p27 and NEDD8 in SI-} NET

Next, we evaluated the connection between $C D K N 1 B / \mathrm{p} 27$ and NEDD8 in two other SI-NET clinical sample cohorts.
In the first cohort (Supplementary Table S10), the mRNA level relationship between $C D K N 1 B$ and $N E D D 8$ was assessed in 62 SI-NET samples from 47 patients using quantitative real-time PCR (qPCR). Supporting the MSbased protein level analysis, a positive correlation was here 
Fig. 6 p27 stability is dependent on NEDD8 and RBX1 levels. a Western blotting showing NEDD8 and p27 levels in response to siRNA-based silencing of NEDD8 in CNDT2 cells. The barplot indicates relative protein levels normalized to GAPDH as determined by densitometry analysis of western blotting results. b Western blotting and barplot showing RBX1 and p27 levels in response to siRNAbased silencing of RBX1 in CNDT2 cells. c Western blotting and barplot showing p27 levels in response to siRNA-based silencing of p27 in CNDT2 cells (left). Right barplot shows flow cytometry analysis of Annexin V and PI staining of CNDT2 cells for evaluation of early apoptosis in response to pevonedistat. Colors indicate whether cells were pretreated with siRNA for silencing of p27 (pink or red) or non-targeting control siRNA (green). Error bars in flow cytometry analysis indicate SEM from six experiments, and $p$-values were calculated using paired $t$-test. d Western blottings and barplot showing p27, NEDD8, RBX1, and cleaved PARP (cPARP) levels in response to siRNA-based silencing of NEDD8/RBX1 in combination or NEDD8/RBX1/p27 in combination in CNDT2 cells. NTC lane in western blotting indicate non-transfected control cells (NTC). All western blotting quantifications were based on three independent experiments with barplot error bars indicating SD and $p$-values calculated by Student's $t$-test

observed between $C D K N 1 B$ and NEDD 8 mRNA levels (Supplementary Fig. S5). This result suggests that there could be a transcriptional feedback mechanism between $C D K N 1 B$ and $N E D D 8$. To investigate potential causes of differential $C D K N 1 B$ expression in SI-NET, we investigated $C D K N 1 B$ copy number alterations and $C D K N 1 B$ promoter methylation. Eight tumors showed copy number loss of $C D K N 1 B$, two tumors had copy number gain, whereas the remaining tumors exhibited two copies. However, no significant association could be found between $C D K N 1 B$ expression and copy number alterations. $C D K N 1 B$ promoter methylation was quantified by $\mathrm{Pyr}-$ osequencing. Low levels of methylation with MetI below $10 \%$ were revealed in 64/65 tumor samples, whereas one single case had an increased MetI of 26\% (Supplementary Table S10). In summary, the differential mRNA expression of $C D K N 1 B$ in SI-NET could not be explained by altered copy number or promotor methylation.

To evaluate protein-level association between NEDD8 and p27, immunohistochemistry (IHC) analysis was employed in a separate cohort of 43 SI-NETs (Supplementary Table S11). NEDD8 and p27 demonstrated a spectrum of none to high cytoplasmic and nuclear staining intensities among different samples (Fig. 7a-d). However, no association between the level of NEDD8 and p27 or with clinicopathological features including presence of liver metastasis was observed. In summary, these results did not validate the initial finding that NEDD8 protein levels were directly coupled to the presence of liver metastasis. The immunohistochemical analysis however did support the differential expression of both NEDD8 and CDKN1B/p27 in SI-NET, indicating that some tumors may be relying on increased CRL activity for growth and survival.

\section{Discussion}

The proteomics profiling here performed indicates overexpression of the CRL regulators NEDD8 and RBX1 in a subset of SI-NETs. Further, the strong correlation between NEDD8 and RBX1 both at protein level, as detected in SINET, and at mRNA level shown by public domain data analysis across different cancer types suggest that these genes are transcriptionally co-regulated. Previous research support a connection between overexpression of these two proteins and cancer. As an example it has been shown that high expression of RBX1 is coupled to poor prognosis in gastric cancer [25], bladder cancer [26], and non-small cell lung cancer [27]. Similarly, NEDD8 overexpression has been coupled to poor prognosis in hepatocellular carcinoma [28] and bladder cancer [29], and increased levels of neddylated Cullin-1 has previously been shown in high-grade lung NETs [30]. Also in nasopharyngeal carcinoma, overexpression of NEDD8 was coupled to shorter overall survival [31] and in the same publication it was also shown in cell line models that suppression of NEDD8 levels directly inhibits cell growth. In addition, enzymes involved in the neddylation process are upregulated in a range of different cancer types [32].

Previous studies have shown that $C D K N 1 B$ stands out so far as the only recurrently mutated gene in SI-NETs, establishing $C D K N 1 B / \mathrm{p} 27$ as a key tumor suppressor in this disease [6]. The relatively low frequency of $C D K N 1 B$ mutations $(<10 \%)$ however suggests that additional, so far unknown, mechanisms to inactivate tumor suppressors exist in SI-NET. The data presented here show considerable heterogeneity between individual SI-NETs in expression of $C D K N 1 B / \mathrm{p} 27$, both at the mRNA and at the protein level. At the mRNA level, these differences could not be directly attributed to copy number differences or epigenetic regulation by methylation. This would suggest that other mechanisms such as transcriptional and posttranscriptional regulation affect the mRNA level of $C D K N 1 B$ in SI-NET. Intriguingly, we did detect an association between the mRNA level of $C D K N 1 B$ and the mRNA level of NEDD 8 in SI-NET. This finding could indicate that a negative feedback mechanism exists at the transcriptional level to limit the inhibitory effect of p27 on cell cycle entry. Importantly, inhibition of neddylation resulted in stabilization of p27 in all four NET cell lines examined, clearly showing that p27 levels are controlled at the posttranslational level in NET. Further, silencing of either NEDD8 or RBX1 resulted in stabilization of $\mathrm{p} 27$, indicating that the level of these proteins directly impacts on CRL activity and stability of substrates such as p27. Our data thus suggest that in a subgroup of SI-NETs, an additional mechanism to overcome the tumor suppressor function of $C D K N 1 B / \mathrm{p} 27$ could be through increased CRL activity resulting in 
Fig. 7 Clinical evaluation of NEDD8 and CDKN1B/p27. a Overview of immunohistochemistry analysis for NEDD8 and p27 in 43 primary SI-NETs is shown. b-d Photomicrographs exemplifying diverse cytoplasmic and nuclear intensity of NEDD8 and p27 immunoreactivity in SI-NETs. b Case IHC-7 demonstrating strong cytoplasmic and nuclear NEDD8 (3+) and weak cytoplasmic and nuclear p27 $(1+)$ staining, respectively. c Case IHC-18 exhibiting weak NEDD8 (1+) and strong p27 immunoreactivity. d Case IHC-5 with strong staining $(3+)$ for both proteins. Cytoplasmic and nuclear staining were intimately associated and therefore scored conjoined. Membranous staining patterns were not observed. All photomicrographs are magnified $\times 100$ a

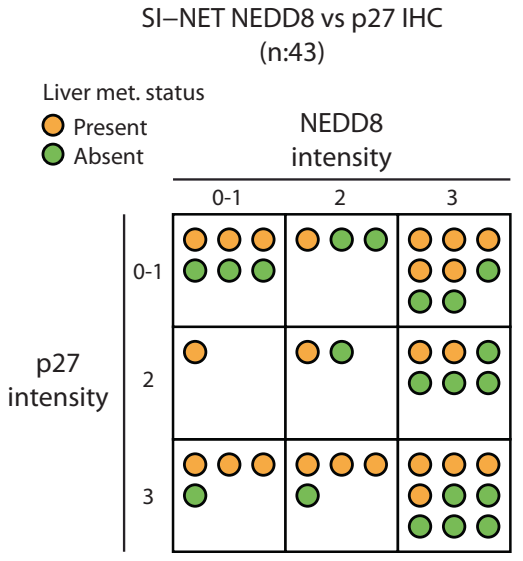

C

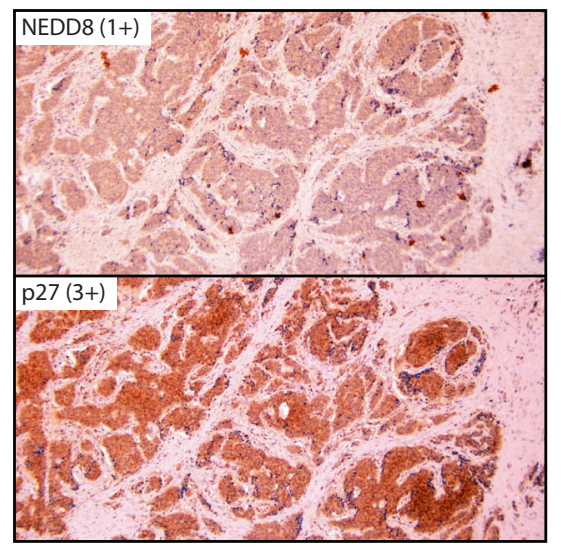

b

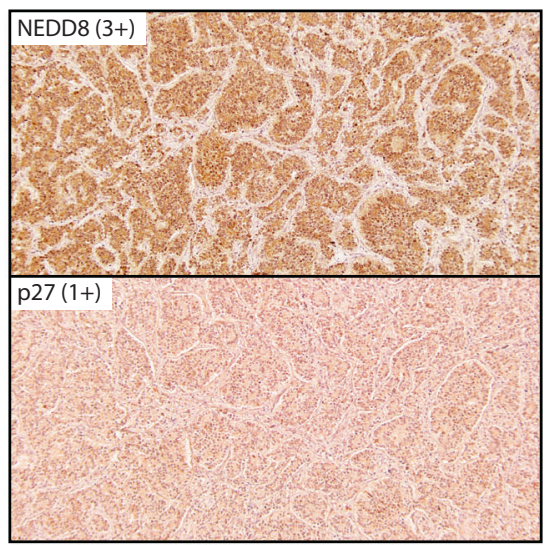

d

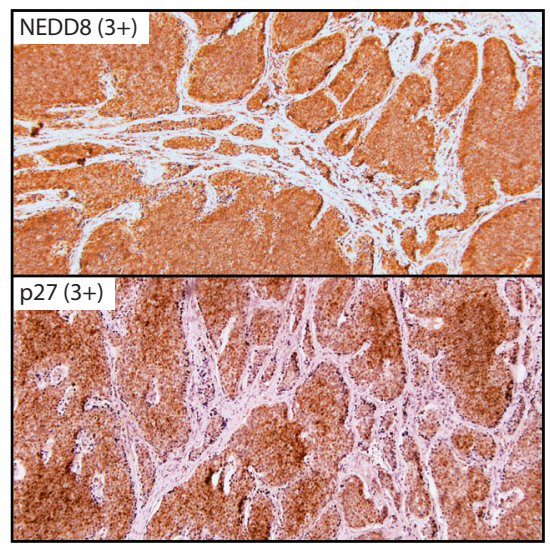

degradation of $\mathrm{p} 27$. Deregulation of $C D K N 1 B / \mathrm{p} 27$ has been reported in many cancer types [33,34], but mutations are rare and loss of heterozygosity with total loss of expression is not observed in cancer [35]. Several studies have shown that complete loss of p27 would result in abrogation of cell proliferation, whereas expression levels lower than normal can lead to increased cyclin-dependent kinase activity and subsequent cell proliferation $[36,37]$. Based on the recurrent heterozygous inactivating mutations in SI-NET, $C D K N 1 B$ is considered a haploinsufficient tumor suppressor gene. In other words, the expression of $C D K N 1 B$ from a single copy is insufficient to sustain a tumor suppressor function. A similar net effect could be accomplished in non-mutated tumors by increasing the proteasomal degradation of p27 through activation of CRLs.

Even though we were unable to establish a direct relationship between NEDD8 expression and SI-NET progression or metastasis, our data point out inhibition of neddylation as a potential therapeutic strategy in a subset of SI-NETs. In patients with tumors showing reduced level of p27, either through heterozygous mutations or through augmented proteasomal degradation, increasing the levels of p27 through inhibition of its degradation could prove to be a successful treatment strategy.

Targeting the neddylation pathway as a therapeutic strategy for cancer treatment was first demonstrated by the development of the NAE inhibitor pevonedistat [20]. Pevonedistat was shown to inhibit cullin neddylation, resulting in a concomitant stabilization of a number of known CRL substrates including p27, Nrf2, and CDT1, loss of cell cycle control, and induction of apoptosis. It was speculated that the specific cell death mechanism was at least in part due to CDT1-stimulated re-replication without cell cycle progression, ultimately triggering apoptosis. As all cullins are neddylated by NAE, it is expected that all CRL complexes would be inhibited by pevonedistat, whereas other, non-CRL E3 ligases, would remain active. It was estimated by a protein turnover analysis comparing bortezomib with pevonedistat that $\sim 20 \%$ of the proteasome-dependent degradation is mediated by CRL-dependent ubiquitination [20]. The molecular mechanism of action for pevonedistat was later described, as it was shown that in the presence of pevonedistat, NAE catalyzes the formation of a NEDD8-pevonedistat adduct. The stability of this adduct within the active site of 
NAE effectively and specifically blocks further NAE enzymatic activity [38]. Pevonedistat has recently been evaluated in phase I trials for treatment of different cancer types such as myeloma/lymphoma [39], AML [40], melanoma [41], and other solid tumors [42], in all instances showing that pevonedistat in general was well tolerated at doses tested. To our knowledge, this is the first report describing the use of pevonedistat in neuroendocrine cells. Importantly, we show here that SI-NET cell lines are sensitive to CRL inhibition by both NEDD8/RBX1 silencing and pharmacological inhibition of the neddylation machinery using pevonedistat. Our data also show that part of the cellular effects of CRL inhibition is p27 dependent, as silencing of p27 resulted in reduced apoptosis.

Further, proteomics time-course profiling of pevonedistat effects in two different SI-NET cell lines revealed rapid stabilization of multiple proteins, in line with the anticipated effects of CRL inhibition. Additional proteomics profiling of cellular response to proteasomal inhibition using bortezomib allowed us to nominate a short list of candidate CRL substrates in SI-NET. Among the stabilized proteins were several well-known CRL substrates as well as canonical tumor suppressors with potential importance in SI-NET. Even though we have focused our attention to p27 as an important CRL target in SI-NET, our analysis identified additional proteins that were stabilized in response to pevonedistat. Anyone of these proteins or a cohort of them could contribute to the effects of pevonedistat treatment in SI-NET. These results could prove important in the future work to identify predictive markers for pevonedistat treatment.

In summary, our study supports the significance of the CDKN1B/p27 in SI-NET and identifies CRL-dependent proteasomal degradation as an additional mechanisms for inactivation of this tumor suppressor in SI-NET. Based on this finding we evaluated and showed neddylation as a potential new drug target in SI-NET cells using the targeted compound pevonedistat. Future studies are needed to evaluate the use of pevonedistat in a clinical setting as well as to identify predictive biomarkers in SI-NET.

\section{Materials and methods}

\section{Cell lines}

Four NET cell lines were used (BON-1, NCI-727, HC45, and CNDT2). Sources, verifications, and culturing conditions are given in Supplementary Methods.

\section{Tissue samples}

All tissue samples were obtained from the Karolinska University Hospital biobank and had been collected with informed patients' consent and approval from the local ethics committee in Stockholm (Dnr:2012/305-31/1). Fourteen tumors (Screen-1 to -14; Supplementary Table S1) were used for proteomics. Another 70 tumors (case numbers 1-70; Supplementary Table S10) from 50 SI-NET patients were used for pyrosequencing and qPCR analyses. An additional cohort of 43 paraffin-embedded tumors (IHC-1 to -43; Supplementary Table S11) was used for IHC. Adjacent normal tissues from 12 patients served as non-tumor references. Further descriptions are given in Supplementary Methods.

\section{Proteomics analysis using HiRIEF-LC-MS/MS}

Protein expression profiles were generated using the HiRIEF-LC-MS/MS methodology [43] and procedures detailed in refs. [44] and [45]. In the first proteomics analysis, primary SI-NETs from seven patients with liver metastases and from seven patients without detectable liver metastases at the time of surgery were included. In the second proteomics analysis, CNDT2 and HC45 cells treated with $400 \mathrm{nM}$ pevonedistat for $3,6,12$, or $24 \mathrm{~h}$ as well as dimethylsulfoxide (DMSO)-treated control cells were analyzed in duplicates. In the third proteomics analysis, CNDT2 cells treated with $500 \mathrm{nM}$ bortezomib (triplicate), $500 \mathrm{nM}$ bortezomib plus $500 \mathrm{nM}$ pevonedistat (triplicate) for $12 \mathrm{~h}$, as well as DMSO-treated control cells (four replicates) were analyzed. The MS proteomics data have been deposited to the ProteomeXchange Consortium via the PRIDE partner repository with the dataset identifier PXD009877 and PXD013024. Experimental procedures and data analyses are further described in Supplementary Methods.

\section{Proliferation assay}

For the proliferation assay, BrdU proliferation kit (Roche) was used following the manufacturer's instructions. Briefly, cells were seeded in 96-well plates and treated with pevonedistat at concentration $0,100,200$, $400,600,800,1000$, and $2000 \mathrm{nM}$ for 3 days. BrdU reagent was added to the medium for a period of $4 \mathrm{~h}$ after which cells were fixed and incubated with the BrdU antibody for $90 \mathrm{~min}$. The intensity of the colorimetric reaction, which is proportional to the incorporated $\mathrm{BrdU}$, was measured at the wavelength $370 \mathrm{~nm}$ and the reference wavelength of $492 \mathrm{~nm}$ using a microplate ELISA reader (VERSAmax; Molecular Devices, Sunnyvale, CA, USA). Curve fitting and generation of dose-response figures was performed in $\mathrm{R}$ using the drc package [46] with standard settings using the four-parameter log-logistic model function (LL.4). IC $_{50}$ values were then retrieved from the fitted curve. 


\section{Flow cytometry assessment of apoptosis}

Apoptosis assays were performed based on redistribution of phosphatidylserine phospholipid components of the cell membrane and a high affinity of Annexin $\mathrm{V}$ fluorescein (FLUOS) toward phosphatidylserine. BON-1, CNDT2, HC45, and NCI-H727 cells were treated with DMSO as a control or pevonedistat at the concentrations of 500 and $1000 \mathrm{nM}$ in three wells of a six-well plate for 3 days. After collection of cells by trypsination and washing twice in cold phosphate-buffered saline, cells were incubated for $15 \mathrm{~min}$ in fluorescence-activated cell sorting (FACS) buffer $(10 \mathrm{mM}$ HEPES/NaOH pH 7.4, $140 \mathrm{mM} \mathrm{NaCl}, 5 \mathrm{mM}$ $\mathrm{CaCl}_{2}$ ) containing $20 \mu \mathrm{l}$ Annexin $\mathrm{V}$ and $20 \mu \mathrm{l}$ propidium iodide $(50 \mu \mathrm{M})$ added per $\mathrm{ml}$ buffer. Thereafter, $400 \mu \mathrm{L}$ of FACS buffer was added to the cells and analysis was performed using NovoCyte flow cytometer (ACEA Biosciences, San Diego, CA, USA) following the manufacturer's instructions (Roche 11828681001). For the analysis of four different NET cell lines, the mean of data from at least three independent biological replications were used to create the graphs (Fig. 4b). For the siRNA experiment, CNDT2 cells were first transfected with either an siRNA targeting p27 or a negative control siRNA. Twentyfour hours after transfection, cells were treated with either DMSO or $500 \mathrm{nM}$ pevonedistat for $24 \mathrm{~h}$ before analysis. Staining was carried out as described above and the FACS analysis was carried out on a Fortessa flow cytometer (BD Biosciences, San Jose, CA, USA). The mean of the data from at least six independent experiments was used to create the graphs.

\section{siRNA-based silencing}

siRNA treatments of CNDT2 cells were performed according to standard protocol. Briefly, RNAiMAX transfection reagent (Invitrogen) and siRNA (two siRNA each for RBX1, NEDD8, CDKN1B, and negative control; Qiagen) were diluted separately in Opti-MEM medium (ThermoFisher), mixed (1:1 ratio), and incubated for 5 min. RNA-lipid complexes were then added to cells in six-well plates, seeded to be $60 \%$ confluent at transfection, and cells were then incubated for $1-3$ days at $37^{\circ} \mathrm{C}$ before analysis.

\section{Western blotting analyses}

Proteins were extracted from the cell lines, separated by SDS-polyacrylamide gel electrophoresis and transferred to nitrocellulose or polyvinylidene difluoride membranes (Invitrogen). After blocking in albumin or milk, membranes were incubated at $4{ }^{\circ} \mathrm{C}$ overnight with primary antibodies for NEDD8 (19E3, Cell Signaling) at dilution 1:1000, RBX1 (D3J5I, Cell Signaling) at 1:1000, cPARP (E51, Abcam) at 1:10,000, p27 (D69C12, Cell Signaling) at 1:1000, p27 (sc-528, Santa Cruz) at 1:500, Cullin 1 (Santa Cruz 1:1000), and GAPDH (sc-47724, Santa Cruz) at 1:10,000). GAPDH was used as a control of loading and transfer. Each experiment was biologically repeated at least three times.

\section{DNA copy number, gene expression, and methylation analyses}

DNA copy numbers were determined by qPCR for CDKN1B (Hs02134847_cn, Applied Biosystems) and the endogenous control RNaseP (\#4403326, ThermoFisher Scientific) according to the methodology described in ref. [47]. Gene expressions were quantified by TaqMan for CDKN1B (Hs01597588_m1, Applied Biosystems) and NEDD8 (Hs04187792_m1) with the endogenous control ACTB (Hs99999903_m1). DNA methylation was quantified for the $C D K N 1 B$ promoter (Hs_CDKN1B_01_PM, Qiagen) using pyrosequencing. For further details and experimental procedures, see Supplementary Methods.

\section{Immunohistochemistry}

IHC was performed on the 43 SI-NET tissue samples for NEDD8 (Cell Signaling) and p27 (Cell Signaling, D69C12) as detailed in Supplementary Methods. A combined score of nuclear and cytoplasmic staining was obtained: 0 (absent), 1 (low), 2 (medium), and 3 (high).

\section{Statistical analyses}

Statistical comparisons were done using Student's $t$-test, Fisher's exact test, Mann-Whitney $U$-test, Pearson's or Spearman's correlation. Details are given in Supplementary Methods.

Acknowledgements The study was supported by grants from Novartis, the Swedish Research Council, the Swedish Cancer Society, the Cancer Society in Stockholm, the Gustav V Jubilee Foundation, Stockholm County Council, and Karolinska Institutet. Mass spectrometry-based proteomics analysis was performed at the Clinical Proteomics Mass Spectrometry facility located at SciLifeLab in Solna, Sweden.

\section{Compliance with ethical standards}

Conflict of interest The authors declare that they have no conflict of interest.

Publisher's note: Springer Nature remains neutral with regard to jurisdictional claims in published maps and institutional affiliations. 
Open Access This article is licensed under a Creative Commons Attribution 4.0 International License, which permits use, sharing, adaptation, distribution and reproduction in any medium or format, as long as you give appropriate credit to the original author(s) and the source, provide a link to the Creative Commons license, and indicate if changes were made. The images or other third party material in this article are included in the article's Creative Commons license, unless indicated otherwise in a credit line to the material. If material is not included in the article's Creative Commons license and your intended use is not permitted by statutory regulation or exceeds the permitted use, you will need to obtain permission directly from the copyright holder. To view a copy of this license, visit http://creativecommons. org/licenses/by/4.0/.

\section{References}

1. Stalberg P, Westin G, Thirlwell C. Genetics and epigenetics in small intestinal neuroendocrine tumours. J Intern Med. 2016;280:584-94.

2. Capella C, Arnold R, Klimstra DS, Klöppel G, Komminoth P, Solcia E, Rindi G. Neuroendocrine neoplasms of the small instestine. In: Bosman FT, Carneiro F, Hruban RH, Theise ND (eds). WHO classification of tumours of the digestive system. 4th edn. (International Agency for Research on Cancer, Lyon, 2010) pp 102-107.

3. Cunningham JL, Janson ET. The biological hallmarks of ileal carcinoids. Eur J Clin Invest. 2011;41:1353-60.

4. Kytola S, Hoog A, Nord B, Cedermark B, Frisk T, Larsson C, et al. Comparative genomic hybridization identifies loss of 18q22qter as an early and specific event in tumorigenesis of midgut carcinoids. Am J Pathol. 2001;158:1803-8.

5. Nieser M, Henopp T, Brix J, Stoss L, Sitek B, Naboulsi W, et al. Loss of Chromosome 18 in neuroendocrine tumors of the small intestine: the enigma remains. Neuroendocrinology. 2017;104:302-12.

6. Francis JM, Kiezun A, Ramos AH, Serra S, Pedamallu CS, Qian ZR, et al. Somatic mutation of CDKN1B in small intestine neuroendocrine tumors. Nat Genet. 2013;45:1483-6.

7. Karpathakis A, Dibra H, Pipinikas C, Feber A, Morris T, Francis $\mathrm{J}$, et al. Prognostic impact of novel molecular subtypes of small intestinal neuroendocrine tumor. Clin Cancer Res. 2016;22:250-8.

8. Pagano M, Tam SW, Theodoras AM, Beer-Romero P, Del Sal G, Chau V, et al. Role of the ubiquitin-proteasome pathway in regulating abundance of the cyclin-dependent kinase inhibitor p27. Science. 1995;269:682-5.

9. Carrano AC, Eytan E, Hershko A, Pagano M. SKP2 is required for ubiquitin-mediated degradation of the CDK inhibitor p27. Nat Cell Biol. 1999;1:193-9.

10. Zheng N, Schulman BA, Song L, Miller JJ, Jeffrey PD, Wang P, et al. Structure of the Cul1-Rbx1-Skp1-F boxSkp2 SCF ubiquitin ligase complex. Nature. 2002;416:703-9.

11. Loda M, Cukor B, Tam SW, Lavin P, Fiorentino M, Draetta GF, et al. Increased proteasome-dependent degradation of the cyclindependent kinase inhibitor p27 in aggressive colorectal carcinomas. Nat Med. 1997;3:231-4.

12. Frescas D, Pagano M. Deregulated proteolysis by the F-box proteins SKP2 and beta-TrCP: tipping the scales of cancer. Nat Rev Cancer. 2008;8:438-49.

13. Pan ZQ, Kentsis A, Dias DC, Yamoah K, Wu K. Nedd8 on cullin: building an expressway to protein destruction. Oncogene. 2004;23:1985-97.

14. Enchev RI, Schulman BA, Peter M. Protein neddylation: beyond cullin-RING ligases. Nat Rev Mol Cell Biol. 2015;16:30-44.

15. Duda DM, Borg LA, Scott DC, Hunt HW, Hammel M, Schulman BA. Structural insights into NEDD8 activation of cullin-RING ligases: conformational control of conjugation. Cell. 2008;134:995-1006.

16. Gao J, Chen T, Liu J, Liu W, Hu G, Guo X, et al. Loss of NECL1, a novel tumor suppressor, can be restored in glioma by HDAC inhibitor-Trichostatin A through Sp1 binding site. Glia. 2009;57:989-99.

17. Edfeldt K, Daskalakis K, Backlin C, Norlen O, Tiensuu Janson E, Westin G, et al. DcR3, TFF3, and midkine are novel serum biomarkers in small intestinal neuroendocrine tumors. Neuroendocrinology. 2017;105:170-81.

18. Watson IR, Irwin MS, Ohh M. NEDD8 pathways in cancer, Sine Quibus Non. Cancer Cell. 2011;19:168-76.

19. Cancer Genome Atlas Research N, Weinstein JN, Collisson EA, Mills GB, Shaw KR, Ozenberger BA, et al. The Cancer Genome Atlas Pan-Cancer analysis project. Nat Genet. 2013;45:1113-20.

20. Soucy TA, Smith PG, Milhollen MA, Berger AJ, Gavin JM, Adhikari S, et al. An inhibitor of NEDD8-activating enzyme as a new approach to treat cancer. Nature. 2009;458:732-6.

21. Yang W, Soares J, Greninger P, Edelman EJ, Lightfoot H, Forbes $\mathrm{S}$, et al. Genomics of Drug Sensitivity in Cancer (GDSC): a resource for therapeutic biomarker discovery in cancer cells. Nucleic Acids Res. 2013;41:D955-961.

22. Galvan JA, Astudillo A, Vallina A, Fonseca PJ, Gomez-Izquierdo L, Garcia-Carbonero R, et al. Epithelial-mesenchymal transition markers in the differential diagnosis of gastroenteropancreatic neuroendocrine tumors. Am J Clin Pathol. 2013;140:61-72.

23. Emanuele MJ, Elia AE, Xu Q, Thoma CR, Izhar L, Leng Y, et al. Global identification of modular cullin-RING ligase substrates. Cell. 2011;147:459-74.

24. Futreal PA, Coin L, Marshall M, Down T, Hubbard T, Wooster R, et al. A census of human cancer genes. Nat Rev Cancer. 2004;4:177-83.

25. Migita K, Takayama T, Matsumoto S, Wakatsuki K, Tanaka T, Ito $\mathrm{M}$, et al. Prognostic impact of RING box protein-1 (RBX1) expression in gastric cancer. Gastric Cancer. 2014;17:601-9.

26. Wang W, Qiu J, Liu Z, Zeng Y, Fan J, Liu Y, et al. Overexpression of RING box protein-1 (RBX1) associated with poor prognosis of non-muscle-invasive bladder transitional cell carcinoma. J Surg Oncol. 2013;107:758-61.

27. Xing R, Chen KB, Xuan Y, Feng C, Xue M, Zeng YC. RBX1 expression is an unfavorable prognostic factor in patients with non-small cell lung cancer. Surg Oncol. 2016;25:147-51.

28. Yu J, Huang WL, Xu QG, Zhang L, Sun SH, Zhou WP, et al. Overactivated neddylation pathway in human hepatocellular carcinoma. Cancer Med. 2018. (PMID: 29846044).

29. Tian DW, Wu ZL, Jiang LM, Gao J, Wu CL, Hu HL. Neural precursor cell expressed, developmentally downregulated 8 promotes tumor progression and predicts poor prognosis of patients with bladder cancer. Cancer Sci. 2019;110:458-67.

30. Salon C, Brambilla E, Brambilla C, Lantuejoul S, Gazzeri S, Eymin B. Altered pattern of Cul-1 protein expression and neddylation in human lung tumours: relationships with CAND1 and cyclin E protein levels. J Pathol. 2007;213:303-10.

31. Xie P, Yang JP, Cao Y, Peng LX, Zheng LS, Sun R, et al. Promoting tumorigenesis in nasopharyngeal carcinoma, NEDD8 serves as a potential theranostic target. Cell Death Dis. 2017;8:e2834.

32. Zhou L, Zhang W, Sun Y, Jia L. Protein neddylation and its alterations in human cancers for targeted therapy. Cell Signal. 2018;44:92-102.

33. Chu IM, Hengst L, Slingerland JM. The Cdk inhibitor p27 in human cancer: prognostic potential and relevance to anticancer therapy. Nat Rev Cancer. 2008;8:253-67.

34. Philipp-Staheli J, Payne SR, Kemp CJ. p27(Kip1): regulation and function of a haploinsufficient tumor suppressor and its misregulation in cancer. Exp Cell Res. 2001;264:148-68. 
35. Lee J, Kim SS. The function of p27 KIP1 during tumor development. Exp Mol Med. 2009;41:765-71.

36. Gao H, Ouyang X, Banach-Petrosky W, Borowsky AD, Lin Y, Kim M, et al. A critical role for p27kip1 gene dosage in a mouse model of prostate carcinogenesis. Proc Natl Acad Sci USA. 2004;101:17204-9.

37. Muraoka RS, Lenferink AE, Law B, Hamilton E, Brantley DM, Roebuck LR, et al. ErbB2/Neu-induced, cyclin D1-dependent transformation is accelerated in p27-haploinsufficient mammary epithelial cells but impaired in p27-null cells. Mol Cell Biol. 2002;22:2204-19.

38. Brownell JE, Sintchak MD, Gavin JM, Liao H, Bruzzese FJ, Bump NJ, et al. Substrate-assisted inhibition of ubiquitin-like protein-activating enzymes: the NEDD8 E1 inhibitor MLN4924 forms a NEDD8-AMP mimetic in situ. Mol Cell. 2010;37:102-11.

39. Shah JJ, Jakubowiak AJ, O'Connor OA, Orlowski RZ, Harvey $\mathrm{RD}$, Smith MR, et al. Phase I study of the novel investigational NEDD8-activating enzyme inhibitor pevonedistat (MLN4924) in patients with relapsed/refractory multiple myeloma or lymphoma. Clin Cancer Res. 2016;22:34-43.

40. Swords RT, Coutre S, Maris MB, Zeidner JF, Foran JM, Cruz J, et al. Pevonedistat, a first-in-class NEDD8-activating enzyme (NAE) inhibitor, combined with azacitidine, in patients with AML. Blood. 2018;131:1415-24.

41. Bhatia S, Pavlick AC, Boasberg P, Thompson JA, Mulligan G, Pickard MD, et al. A phase I study of the investigational NEDD8- activating enzyme inhibitor pevonedistat (TAK-924/MLN4924) in patients with metastatic melanoma. Invest New Drugs. 2016;34:439-49.

42. Sarantopoulos J, Shapiro GI, Cohen RB, Clark JW, Kauh JS, Weiss GJ, et al. Phase I study of the investigational NEDD8activating enzyme inhibitor pevonedistat (TAK-924/MLN4924) in patients with advanced solid tumors. Clin Cancer Res. 2016;22:847-57.

43. Branca RM, Orre LM, Johansson HJ, Granholm V, Huss M, Perez-Bercoff A, et al. HiRIEF LC-MS enables deep proteome coverage and unbiased proteogenomics. Nat Methods. 2014;11:59-62.

44. Zhou Y, Frings O, Branca RM, Boekel J, le Sage C, Fredlund E, et al. microRNAs with AAGUGC seed motif constitute an integral part of an oncogenic signaling network. Oncogene. 2017;36:731-45.

45. Fotouhi O, Kjellin H, Larsson C, Hashemi J, Barriuso J, Juhlin $\mathrm{CC}$, et al. Proteomics suggests a role for APC-survivin in response to somatostatin analog treatment of neuroendocrine tumors. J Clin Endocrinol Metab. 2016;101:3616-27.

46. Ritz C, Baty F, Streibig JC, Gerhard D. Dose-response analysis using R. PLoS ONE. 2015;10:e0146021

47. Hashemi J, Fotouhi O, Sulaiman L, Kjellman M, Hoog A, Zedenius $\mathrm{J}$, et al. Copy number alterations in small intestinal neuroendocrine tumors determined by array comparative genomic hybridization. BMC Cancer. 2013;13:505. 